\title{
Conditional Knock-out of mGluR5 from Astrocytes during Epilepsy Development Impairs High-Frequency Glutamate Uptake
}

\author{
(DAnthony D. Umpierre, ${ }^{1,2}$ ๑Peter J. West, ${ }^{1,2,3}$ ๑John A. White, ${ }^{4}$ and $\odot$ Karen S. Wilcox ${ }^{1,2,3}$ \\ ${ }^{1}$ Interdepartmental Program in Neuroscience, ${ }^{2}$ Department of Pharmacology \& Toxicology, ${ }^{3}$ Anticonvulsant Drug Development Program, University of \\ Utah, Salt Lake City, Utah 84112, and ${ }^{4}$ Department of Biomedical Engineering, Boston University, Boston, Massachusetts 02215
}

Astrocyte expression of metabotropic glutamate receptor 5 (mGluR5) is consistently observed in resected tissue from patients with epilepsy and is equally prevalent in animal models of epilepsy. However, little is known about the functional signaling properties or downstream consequences of astrocyte mGluR5 activation during epilepsy development. In the rodent brain, astrocyte mGluR5 expression is developmentally regulated and confined in expression/function to the first weeks of life, with similar observations made in human control tissue. Herein, we demonstrate that mGluR5 expression and function dramatically increase in a mouse model of temporal lobe epilepsy. Interestingly, in both male and female mice, mGluR5 function persists in the astrocyte throughout the process of epileptogenesis following status epilepticus. However, mGluR5 expression and function are transient in animals that do not develop epilepsy over an equivalent time period, suggesting that patterns of mGluR5 expression may signify continuing epilepsy development or its resolution. We demonstrate that, during epileptogenesis, astrocytes reacquire mGluR5-dependent calcium transients following agonist application or synaptic glutamate release, a feature of astrocyte-neuron communication absent since early development. Finally, we find that the selective and conditional knock-out of mGluR5 signaling from astrocytes during epilepsy development slows the rate of glutamate clearance through astrocyte glutamate transporters under high-frequency stimulation conditions, a feature that suggests astrocyte mGluR5 expression during epileptogenesis may recapitulate earlier developmental roles in regulating glutamate transporter function.

Key words: astrocyte; glutamate transporter; kainate; mGluR5; status epilepticus; temporal lobe epilepsy

Significance Statement

In development, astrocyte mGluR5 signaling plays a critical role in regulating structural and functional interactions between astrocytes and neurons at the tripartite synapse. Notably, mGluR5 signaling is a positive regulator of astrocyte glutamate transporter expression and function, an essential component of excitatory signaling regulation in hippocampus. After early development, astrocyte mGluR5 expression is downregulated, but reemerges in animal models of temporal lobe epilepsy (TLE) development and patient epilepsy samples. We explored the hypothesis that astrocyte mGluR5 reemergence recapitulates earlier developmental roles during TLE acquisition. Our work demonstrates that astrocytes with mGluR5 signaling during TLE development perform faster glutamate uptake in hippocampus, revealing a previously unexplored role for astrocyte mGluR5 signaling in hypersynchronous pathology.

\section{Introduction}

Temporal lobe epilepsy (TLE) is a debilitating, and often treatmentrefractory, disorder characterized by spontaneous seizure generation

\footnotetext{
Received May 6, 2018; revised Nov. 11, 2018; accepted Nov. 18, 2018

Author contributions: A.D.U. wrote the first draft of the paper; A.D.U.,P.J.W., J.A.W., and K.S.W. edited the paper; A.D.U. and K.S.W. designed research;A.D.U. performed research; A.D.U. and P.J.W. analyzed data; A.D.U. and K.S.W. wrote the paper.

This work was supported by National Institute of Neurological Disorders and Stroke F31 Grant NS096959 to A.D.U. and R01 Grant NS078331 to J.A.W. and K.S.W. We thank Ivan Soltesz for helpful comments regarding experimental design and study considerations; Nathan A. Smith for training in two-photon calcium imaging techniques; and Roy M. Smeal for assistance with calcium imaging and single-cell electrophysiology analysis.
}

from within the hippocampus or adjacent structures. Astrocytes provide an indispensable role in regulating neuronal glutamate transmission in the brain (Tanaka et al., 1997), and particularly in the hippocampus, through their glutamate transporter function (Rothstein et al., 1996). We previously demonstrated that hippocampal astrocytes in a rat model of TLE display faster, potentially

The authors declare no competing financial interests.

Correspondence should be addressed to Karen S. Wilcox at karen.wilcox@hsc.utah.edu.

https://doi.org/10.1523/JNEUROSCI.1148-18.2018

Copyright $\odot 2019$ the authors $\quad 0270-6474 / 19 / 390727-16 \$ 15.00 / 0$ 
compensatory, glutamate transport kinetics before the onset of epilepsy (Takahashi et al., 2010) but did not initially determine the underlying mechanism(s).

The glutamate-sensing surface receptor, mGluR5, has been shown to regulate multiple forms of astrocyte-neuron interaction, including aspects of synaptic ensheathment and glutamate transport in early development. In early development, mGluR5dependent calcium signaling promotes astrocyte motility toward developing glutamatergic synapses (Bernardinelli et al., 2014), establishing a tripartite synapse between astrocyte fine processes and neuronal presynaptic and postsynaptic elements. Loss of $\mathrm{IP}_{3}$ dependent calcium activity, a key effector of astrocyte mGluR5 signaling, reduces the number of tripartite synapses formed in the hippocampus, which slows the kinetics of astrocyte glutamate transport (Tanaka et al., 2013). Additionally, mGluR5 signaling in the astrocyte leads to activity-dependent changes in GLT-1 (EAAT2) expression (Higashimori et al., 2013; Morel et al., 2014), the predominant astrocyte glutamate transporter in the mature hippocampus (Schreiner et al., 2014). Finally, highfrequency synaptic glutamate release can activate astrocyte mGluR5 signaling, which acutely facilitates glutamate transporter function (Devaraju et al., 2013). Together, developmental studies strongly suggest that astrocyte mGluR5 activation is a critical regulator of astrocyte glutamate transport in the developing hippocampal network but has not been studied in the context of epileptogenesis.

Assessing astrocyte mGluR5 signaling as a candidate mechanism for transporter regulation during epileptogenesis requires careful consideration of its dynamic expression and functional patterns (Cai et al., 2000; Panatier and Robitaille, 2016). On the one hand, astrocyte mGluR5 expression is typically confined to the first weeks of rodent brain development (Sun et al., 2013; Morel et al., 2014). On the other hand, astrocyte mGluR5 expression is commonly reported in adult animal models of epilepsy (Aronica et al., 2000; Ulas et al., 2000; Ding et al., 2007; Szokol et al., 2015; Umpierre et al., 2016) and human epilepsy samples (Aronica et al., 2001, 2003; Tang et al., 2001; Sukigara et al., 2014). Using immunohistochemistry (IHC) and two-photon (2-P) calcium imaging techniques, we determined that mGluR5 expression/function reemerges on the astrocyte and has the requisite expression patterns to serve as a mediator of astrocyteneuron interactions during epileptogenesis.

Additionally, we investigated how the absence or presence of mGluR5 signaling impacts the kinetics of glutamate transport during epileptogenesis, using a conditional knock-out mouse to selectively remove mGluR5 expression from astrocytes after development. To model epilepsy development, we used a repeated, low-dose kainic acid injection paradigm (intraperitoneal) to induce status epilepticus (KA-SE), a prolonged seizure state that precipitates the potential development of epilepsy. WT astrocytes in epileptogenesis (KA-SE:mGluR $5^{+/+}$) exhibited mGluR5-dependent calcium transients in response to stimulationinduced glutamate release, a feature of astrocyte-neuron communication absent since early development (Porter and McCarthy, 1996; Honsek et al., 2012), and conditionally repressed in mGluR5 knockout astrocytes (KA-SE:mGluR5 ${ }^{-1-}$ ). The selective loss of mGluR5 during epileptogenesis did not alter intrinsic properties of astrocytes or glutamate uptake kinetics during low-frequency activity. However, similar to developmental studies (Tanaka et al., 2013), selective mGluR5 knock-out specifically impaired glutamate uptake during high-frequency stimulation, a situation that may confer a specific risk for seizures. Overall, our findings suggest that astrocyte mGluR5 expression during epileptogenesis reestablishes earlier forms of astrocyte-neuron communication and serves a compensatory role in regulating astrocyte glutamate transport within the hippocampal network.

\section{Materials and Methods}

Animals. Adult male and female mice were used in all experiments. Ages ranged between 8 and 14 weeks at the time of terminal study, reflecting a span of time points used during epilepsy development. All mice had free access to food and water and were maintained on a $12 \mathrm{~h}$ light/dark cycle in temperature- and humidity-controlled rooms. All procedures conformed to the standards of the National Institutes of Health Guide for the care and use of laboratory animals and were approved by the University of Utah's Institutional Animal Care and Use Committee.

For IHC studies (see Fig. 1), we used Cre-negative offspring from constitutive GFAP-Cre (The Jackson Laboratory, stock \#024098) and PC::G5-tdT (The Jackson Laboratory, stock \#024477) crosses. For 2-P time course studies (see Figs. 2, 3), we used Cre-positive siblings from the above cross to label astrocytes with td-Tomato and report calcium activity using the genetically encoded calcium indicator GCaMP5G (Gee et al., 2014). All other studies used conditional knock-out lines.

Establishing mGluR5 conditional knock-out lines. Standard breeding of existing mouse lines was used to establish multitransgenic mouse lines. First, a mouse line was created that would selectively and conditionally remove mGluR5 expression in astrocytes, by breeding $\mathrm{mGluR} 5{ }^{\mathrm{fl} / \mathrm{fl}}$ mice, kindly provided by Dr. Anis Contractor (Northwestern University) (Xu et al., 2009), to either tamoxifen-inducible GFAP-CreER ${ }^{\mathrm{T} 2}$ mice (The Jackson Laboratory, stock \#012849; see Fig. 6 experiments) or recently developed ALDH1L1-CreER ${ }^{\mathrm{T} 2}$ mice, kindly provided by Dr. Baljit Khakh (University of California, Los Angeles; see experiments in Figs. 5, 7, 8). Additionally, a mouse line was created that could report recombination (tdTomato) and calcium activity (GCaMP5G) in cells that lost mGluR5 expression, by breeding mGluR5 ${ }^{\mathrm{f} / \mathrm{fl}}$ mice to PC::G5-tdT mice. After sibling intercross, we obtained littermates containing the mGluR5 ${ }^{\mathrm{w} / \mathrm{w}}$ or mGluR $5^{\mathrm{f} / \mathrm{fl}}$ sequence. mGluR5 ${ }^{\mathrm{w} / \mathrm{w}} ; \mathrm{PC}:: \mathrm{G} 5-\mathrm{tdT}^{+/+}$mice were bred to either mGluR5 $^{\text {w/w }}$;ALDH1L1-CreER ${ }^{\mathrm{T} 2}$-or-mGluR5 ${ }^{\mathrm{w} / \mathrm{w}}$;GFAP-CreER ${ }^{\mathrm{T} 2}$ mice to create mGluR5 ${ }^{\mathrm{w} / \mathrm{w}}$;ALDH1L1-or-GFAP-CreER ${ }^{\mathrm{T} 2}$;PC::G5-tdT ${ }^{+/-}$offspring, with Cre-positive animals used in experiments. Herein, this line is referred to as mGluR $5^{+/+}$. On the other hand, mGluR5 ${ }^{\mathrm{f} / \mathrm{fl}} ; \mathrm{PC}:: \mathrm{G} 5-\mathrm{tdT}^{+/+}$ mice were bred to either mGluR5 ${ }^{\mathrm{f} / \mathrm{f}}$;ALDH1L1-CreER ${ }^{\mathrm{T} 2}$ or mGluR5 $^{\mathrm{f} / \mathrm{f}}$; GFAP-CreER ${ }^{\mathrm{T} 2}$ mice to create mGluR5 ${ }^{\mathrm{f} / \mathrm{fl}}$;ALDH1L1-or-GFAP-CreER ${ }^{\mathrm{T} 2}$; PC::G5-tdT ${ }^{+/-}$offspring, with Cre-positive animals used in experiments. Herein, this line is referred to as mGluR $5^{-1-}$. To induce recombination after early development (at 6-9 weeks of age), three intraperitoneal injections of tamoxifen were administered to mGluR5 ${ }^{+/+}$and mGluR5 $5^{-1-}$ mice at a dose of $150 \mathrm{mg} / \mathrm{kg}$, with each injection separated by $48 \mathrm{~h}$. Tamoxifen (T5648, Sigma-Aldrich) was dissolved in peanut oil $(20 \mathrm{mg} / \mathrm{ml})$. Animals were given a week of recovery time after the final injection before beginning KA-SE experiments.

Cortical EEG implant surgery. For video EEG studies, GFAP-Cre;PC:: G5-tdT mice were implanted with a cortical EEG above the left parietal cortex at 5-8 weeks of age. Under isoflurane anesthesia (4\% induction, $1 \%-2.5 \%$ maintenance), an incision was made across the midline, three small burr holes were drilled in the skull, and a single-channel electrode (Plastics One) was lowered to contact superficial cortex. The electrode was secured in place with Loctite 454 glue against three implanted anchor screws. Animals were given at least 2 weeks to recover. In all other studies, mGluR5 ${ }^{+/+}$and mGluR5 ${ }^{-1-}$ animals were studied 7-9 d after KA-SE, in a period established previously (Umpierre et al., 2016) and by current studies to faithfully represent a seizure-free latent period $(N=0 / 52$ mice developing epilepsy at this early time point; $24 / 7$ cortical EEG with video monitoring)

Low-dose, KA-SE. Kainic acid (Tocris Bioscience) was dissolved in sterile isotonic saline $(2 \mathrm{mg} / \mathrm{ml})$. Kainate was administered (intraperitoneally) at a dose of $7.5 \mathrm{mg} / \mathrm{kg}$ every $30 \mathrm{~min}$ (Tse et al., 2014; Umpierre et al., 2016) until an animal displayed five or more generalized seizures (defined by Racine stage 3-5 criteria) (Racine, 1972). A naive group was established by injecting animals with an equivalent volume $(100 \mu \mathrm{l})$ of sterile, isotonic saline every $30 \mathrm{~min}$. For video EEG studies, cortical EEG 
was recorded 24/7 using EEG100C amplifiers and MP150 digitizing equipment (BioPac) and synchronized with parallel video monitoring (Thomson and White, 2014), beginning $1 \mathrm{~h}$ before KA-SE and ending at the time point in which the animal was studied. Animal allocation to the naive group or a time point following KA-SE was determined randomly at the time of EEG surgery to best distribute sibling littermates across treatments and time points. No antiseizure drug was administered to stop KA-SE in this paradigm.

IHC and colocalization analysis. Fixed tissue was acquired from crenegative, GFAP-Cre;PC::G5-tdT mice at the following time points: 1, 2, or $3 \mathrm{~d}$ after KA-SE (acute period), $7 \mathrm{~d}$ after KA-SE (latent period), and 28 or $35 \mathrm{~d}$ after KA-SE (chronic period). In the chronic period, no animal developed epilepsy $(N=0 / 7)$, so only nonepilepsy data are presented. Immunofluorescent labeling was performed on $20-\mu \mathrm{m}$-thick coronal sections containing the hippocampus, cut on a freezing-stage microtome (Leica SM2010 R). Astrogliosis was assessed as the area of GFAP immunofluorescence in stratum radiatum, detected by a mouse anti-GFAP antibody preconjugated to AlexaFluor-488 (1:500, EMD Millipore, MAB3402X). Colocalization was assessed by determining the area of overlap between mGluR5 and GFAP immunofluorescence using the ImageJ Coloc plugin (Vargas et al., 2013; Umpierre et al., 2016). mGluR5 was detected by a rabbit anti-mGluR5 primary antibody (1:400, EMD Millipore, AB5675) and a goat anti-rabbit AlexaFluor-555 secondary antibody (1:1000, Invitrogen, A21429). Nuclei were counterstained with DAPI $(1 \mu \mathrm{g} / \mathrm{ml})$. A confocal $z$ stack $(10 \mu \mathrm{m} ; 1 \mu \mathrm{m} z$ step $)$ of hippocampal stratum radiatum was acquired at $20 \times$ magnification for quantification and reimaged at $60 \times$ magnification for illustrative purposes (see Fig. 1), using an Olympus FV1000 confocal microscope.

Acute brain slice preparation. Mice were deeply anesthetized with isoflurane. The brain was rapidly removed, and acute coronal brain slices $(400 \mu \mathrm{m})$ containing the hippocampus were cut on a vibratome (Vibratome 3000, Vibratome) in an ice-cold, sucrose protective solution (in mM as follows: 185 sucrose, $2.5 \mathrm{KCl}, 1.2 \mathrm{NaH}_{2} \mathrm{PO}_{4}, 25 \mathrm{NaHCO}_{3}, 25$ glucose, $10 \mathrm{MgSO}_{4}$, and $0.5 \mathrm{CaCl}_{2}$; osmolarity: 295-300 mOsm). Sections were transferred to a recovery chamber containing aCSF (in mM as follows: $26 \mathrm{NaCl}, 2.5 \mathrm{KCl}, 1 \mathrm{NaH}_{2} \mathrm{PO}_{4}, 26 \mathrm{NaHCO}_{3}, 10.5$ glucose, 1.3 $\mathrm{MgSO}_{4}$, and $2 \mathrm{CaCl}_{2}$; osmolarity: 298-304 mOsm). For single-cell electrophysiology experiments (see Figs. 5, 7, 8), sections were incubated in $34^{\circ} \mathrm{C}$ aCSF for $30 \mathrm{~min}$ then allowed to return to room temperature for an additional $30 \mathrm{~min}$. For other experiments (see Figs. 2, 3, 4, 6), sections recovered for $1 \mathrm{~h}$ at room temperature. All solutions were bubbled with $95 \% \mathrm{O}_{2} / 5 \% \mathrm{CO}_{2}$ and titrated to a $\mathrm{pH}$ of $7.35-7.4$. Acute brain slices were then transferred to a submerged recording chamber and continually perfused with $30^{\circ} \mathrm{C}$ aCSF (in-line heater; TC-324C, Warner Instruments) at a rate of $1-3 \mathrm{ml} / \mathrm{min}$ using a gravity-fed perfusion system.

Agonist application and 2-P calcium imaging. Astrocyte calcium responses to focal agonist application were studied in acute brain slices prepared from cre-positive, GFAP-Cre;PC::G5-tdT mice at 1-3 d after KA-SE (acute period), 7-9 d after KA-SE (latent period), or 28-30 d after KA-SE (chronic period). In the chronic period, animals were subdivided based upon the development of epilepsy or nondevelopment of epilepsy using 24/7 video EEG recording. Epilepsy was defined as the presence of one or more spontaneous seizures meeting Racine stage 2-5 motor criteria after KA-SE. Acute slices from naive animals were studied across multiple, age-matched time points. Calcium activity was recorded using a Prairie 2-P microscope (Bruker) using Prairie View software. T-series were acquired at a $1 \mathrm{~Hz}$ frame rate at $512 \times 512$ pixel resolution, using a water-immersion $20 \times$ lens (NA: 0.95, Olympus) with optical zoom (fixed $273 \times 273 \mu \mathrm{m}$ area).

Agonists were dispensed from a glass microcapillary using a Picospritzer III system (Parker Instrumentation). DHPG (300 or $500 \mu \mathrm{M})$, ATP $(500 \mu \mathrm{M})$, and aCSF were focally applied near stratum radiatum astrocytes (td-Tomato label) in separate trials. Agonists were dissolved in daily aCSF containing AlexaFluor-568 dye (Invitrogen A33081) at a final concentration of $30 \mu \mathrm{m}$. The pressure of application was adjusted between 5 and 12 psi ( $200 \mathrm{~ms}$ pulse) to obtain a consistent spread of AlexaFluor-568 dye between trials and animals. Changes in cytosolic calcium levels were reported by GCaMP5G fluorescence. To study calcium responses in an unbiased manner (see Figs. 2-4), the following steps were taken: (1) the investigator was blinded to the identity of the solution being focally applied; and (2) calcium activity was only quantified within a ROI demarcated by AlexaFluor-568 dye spread (“dye-spread ROI"). We first converted GCaMP $5 \mathrm{G}$ calcium fluorescence to $\Delta \mathrm{F} / \mathrm{F}_{0}$ values, then summated $\Delta \mathrm{F} / \mathrm{F}_{0}$ values over a $10 \mathrm{~s}$ period, beginning at the moment of focal application, to obtain an area under the curve evaluation of calcium changes $\left(\Delta \mathrm{F} / \mathrm{F}_{0} \cdot \mathrm{s}\right)$. To obtain the dye-spread ROI, a maximum intensity projection of dye spread over a $7 \mathrm{~s}$ period was subtracted from a still frame image obtained just before dye application (see Fig. 2A) using ImageJ. After all agonists were applied, the experimenter was "unblinded" to agonist identity. If focal DHPG application produced a clear astrocyte calcium response, the specificity of the DHPG calcium response was assessed in a separate set of paired trials in which DHPG $(500 \mu \mathrm{M})$ was applied in the absence and presence of the selective mGluR5 antagonist MPEP (200 $\mu \mathrm{M}$, bath application). In a separate cohort of animals (acute slices prepared 7-9 d after KA-SE), we determined whether astrocyte calcium transients were influenced by retrograde endocannabinoid signaling. DHPG was focally applied $(500 \mu \mathrm{M})$ in the absence or presence of the cannabinoid receptor 1 (CB1R) antagonist SR-141716A (10 $\mu \mathrm{M}$, bath application). Throughout these experiments, the bath aCSF solution contained 500 nм TTX to mitigate neuronal release of active signaling molecules.

Focal DHPG application ( $500 \mu \mathrm{M}$; see Fig. 5) was additionally used to assess whether astrocytes displayed mGluR5-mediated calcium transients in the mGluR5 ${ }^{+1+}$ and mGluR5 ${ }^{-1-}$ conditions to validate the knock-out approach. This pharmacological approach was also used to correctly identify a cell with and without mGluR5-dependent signaling before studying glutamate transporter currents (GTCs) in that cell using single-cell patch-clamp electrophysiology (for rationale, see Results). In these trials, any td-Tomato astrocyte within $40 \mu \mathrm{m}$ of the pipette tip was considered in the analysis, and $\mathrm{a} \geq 0.5 \Delta \mathrm{F} / \mathrm{F}$ calcium change in the soma or major branches was considered as the threshold for a "response" to DHPG. A response needed to be observed in 2 of 3 repeated trials to reduce the possibility of false-positive inclusion. All selective agonists and antagonists were purchased from Tocris Bioscience.

Schaffer collateral stimulation and 2-P imaging. Acute hippocampal brain slices from cre-positive mGluR5 $5^{+/+}$and mGluR5 ${ }^{-1-}$ animals (GFAP-CreER ${ }^{\mathrm{T} 2}$ driver) were prepared in the latent period following KA-SE (day 7-9). Naive animals (saline-injected, either genotype) were also included at an age-matched time point. Neurotransmitter release from the Schaffer collateral pathway was elicited through a nichrome/ formvar bipolar stimulating electrode $(40 \mathrm{~Hz}, 1 \mathrm{~s}$ stimulation) at a current intensity that elicited a half-maximal fEPSP amplitude. Field potentials were recorded by a glass microelectrode placed 150-200 $\mu \mathrm{m}$ from the stimulating electrode. Astrocyte calcium responses to Schaffer collateral neurotransmitter release were first quantified as the average calcium response $\left(\Delta \mathrm{F} / \mathrm{F}_{0} \cdot \mathrm{s}\right)$ among all responding cells. Astrocyte calcium responses were then evaluated in a paired trial in which calcium activity via mGluR5 signaling was blocked by bath application of the mGluR5 antagonist MPEP $(75 \mu \mathrm{M})$. Additionally, fEPSP amplitude during the $40 \mathrm{~Hz}$ stimulation was also analyzed after fEPSPs consistently reached a plateau (average of the fEPSP amplitude in response to stimulations 15-25).

Single-cell electrophysiology. Acute hippocampal slices were prepared in the latent period (7-9 d after KA-SE) from mGluR5 $5^{+/+}$and mGluR5 $^{-1-}$ animals (ALDH1L1-CreER ${ }^{\mathrm{T} 2}$ driver) (Srinivasan et al., 2016), or from age-matched, naive animals (saline-injected, either genotype). Patch pipettes were pulled by a HEKA PIP 6 system from $1.5 \mathrm{~mm}$ OD, thin-walled borosilicate glass (open tip resistance: $3.5-5.5 \mathrm{M} \Omega$ ). Pipettes were filled with an internal solution containing the following (in $\mathrm{mm}$ ): $120 \mathrm{~K}$-gluconate, $20 \mathrm{HEPES}, 10 \mathrm{EGTA}$, and $0.2 \mathrm{Na}-\mathrm{GTP}$, with AlexaFluor-488 dye (10 $\mu \mathrm{M}$; Invitrogen, A33077) added to the internal solution to allow pipette visualization during 2-P imaging. Internal solutions were made daily from stock solutions and titrated to a $\mathrm{pH}$ of 7.3 with $\mathrm{KOH}$ (final osmolarity: 280-290 mOsm). Under 2-P guidance, tdTomato astrocytes in the stratum radiatum were voltage-clamped at -80 $\mathrm{mV}$ in the whole-cell patch configuration. Series resistance was monitored through $5 \mathrm{mV}$ test pulse application between recordings. Recordings were discontinued if series resistance changed by $>20 \%$, or if resting 
membrane potential (monitored in current-clamp mode, where $\mathrm{I}_{\text {hold }}=$ 0 ) decreased by $>5 \mathrm{mV}$. To pharmacologically isolate GTCs during synaptic activation, the following antagonists were added to the bath aCSF (in $\mu \mathrm{M}$ as follows): $20 \mathrm{CNQX}, 100 \mathrm{D}-\mathrm{AP} 5,100$ picrotoxin, and $200 \mathrm{BaCl}_{2}$ (Bergles and Jahr, 1997). Synaptic activation was achieved through bipolar stimulation of the Schaffer collateral pathway using a constant current stimulator (A385, World Precision Instruments). Recorded astrocytes were $100-200 \mu \mathrm{m}$ from the bipolar stimulating electrode. Whole-cell currents were obtained through a Multiclamp 700A amplifier, a Digidata 1440A board, and pClamp10 software (Molecular Devices). Recordings were sampled at $10 \mathrm{kHz}$ and low-pass filtered at $2 \mathrm{kHz}$. Electrophysiological recordings were analyzed in ClampFit. To determine rates of decay, we fit the decay slope with a first-order time constant $(\tau)$, following either single-pulse stimulation $\left(\tau_{1}\right)$ or the 10th stimulus in a $100 \mathrm{~Hz}$ train $\left(\tau_{10}\right)$. To better visualize decay kinetics after $100 \mathrm{~Hz}$ stimulation (see Fig. $8 \mathrm{C}$ ), we performed subtraction techniques, similar to Diamond and Jahr (2000), to isolate the 10th stimulus trace.

Experimental design and statistical analyses. All statistical analyses were performed in Prism version 7 (GraphPad). All distributions presented display the mean \pm SEM. For most studies, a one-way ANOVA was used to determine group differences. For time course studies (see Figs. 1, 3), a Dunnett's post hoc comparison with naive (see Fig. 1) or inert aCSF (see Fig. 3) was used to determine individual group differences. For selective knock-out studies (see Figs. 6, 7, 8), Tukey's post hoc testing was used to determine differences between three independent groups (naive astrocytes, or astrocytes with or without mGluR5 function during epilepsy development). To characterize an unbiased approach for calcium imaging analysis (see Fig. 2), a two-way ANOVA design was used to evaluate a main effect of agonist, group, and interaction. A Sidak's post hoc test was used to determine individual group differences. The $p$ value reported from one- or two-way ANOVA post hoc testing is adjusted for multiple comparisons. Comparisons of a biological response in the presence or absence of an antagonist used a within-trial design (a two-tailed, paired $t$ test). In one instance (colocalization of mGluR5 and GFAP; see Fig. 1), significant differences between group SDs necessitated a conversion of the colocalized areas to $\log _{10}$ values to restore equal variances and meet criteria for a one-way ANOVA design. The test statistic, degrees of freedom (if applicable), and exact $p$ value are reported in the text. The sample size ( $N$ value always refers to animal number) is reported in the text and figure legends. Significant differences between groups are set at $p<0.05$.

\section{Results \\ Following KA-SE, astrocytes express mGluR5 during a period of peak gliosis}

We used cohorts of male and female, GFAP-Cre;PC::G5-tdT mice (all cre-negative) in IHC experiments to study how astrogliosis (GFAP area) and mGluR5 colocalization changes during phases of epileptogenesis. Epileptogenesis was studied following systemic KA-SE, during an acute period (24-48 h, $N=7$ animals; $72 \mathrm{~h}, N=4)$, a latent period ( $7 \mathrm{~d}, N=6$ ), and a chronic period (28-35d, $N=7$ ). Video EEG (24/7 recording) was used to confirm inclusion of nonepileptic animals in the latent period and to determine whether animals developed epilepsy in the chronic period. All IHC results were compared with agematched, naive tissue $(N=5)$. Following KA-SE, astrocytes become hypertrophied in the hippocampus, with GFAP levels increasing $53 \%$ over a $24-48 \mathrm{~h}$ period, and up to $120 \%$ by $72 \mathrm{~h}$ after KA-SE $\left(F_{(4,24)}=16.37\right.$, one-way ANOVA with Dunnett's post hoc comparison with naive; naive vs $24-48 \mathrm{~h}$ : $p=0.0286$; naive vs $72 \mathrm{~h}: p<0.0001$; Fig. $1 A, B)$. Accompanying peak gliosis $72 \mathrm{~h}$ after KA-SE, we observe strong colocalization of mGluR5 protein with the GFAP-labeled soma and major branches of an astrocyte $\left(F_{(4,24)}=23.65\right.$, one-way ANOVA with Dunnett's post hoc test, $p<0.0001$ vs naive; Fig. $1 A, C)$. Both astrocyte hypertrophy and mGluR5 colocalization are maintained in the seizurefree latent period of the KA-SE model, studied $7 \mathrm{~d}$ after KA-SE (hypertrophy: $F_{(4,24)}=16.37, p=0.0001$ vs naive; colocalization: $F_{(4,24)}=23.65, p<0.0001$ vs naive; one-way ANOVA with Dunnett's post hoc test; Fig. $1 A-C)$. Colocalization patterns suggest that mGluR5 expression can be an early component of reactive astrogliosis, developing soon after KA-SE. However, 28-35 d after KA-SE, animals that did not develop epilepsy no longer displayed either clear astrocyte hypertrophy or mGluR5 colocalization in the hippocampus (hypertrophy: $F_{(4,24)}=16.37, p=$ 0.9988 vs naive; colocalization: $F_{(4,24)}=23.65, p=0.3079$ vs naive; one-way ANOVA with Dunnett's post hoc test; Figure $1 A-$ $C)$. Together, immunofluorescence studies suggest that astrocyte mGluR5 expression is an acute response to KA-SE, which accompanies dynamic changes in astrogliosis. Antibody omission controls do not suggest that staining patterns are the result of antibody cross-reactivity (Fig. $1 D, E$ ).

\section{Astrocyte mGluR5 activation can trigger calcium signaling throughout the process of epileptogenesis}

We next investigated patterns of agonist-induced calcium activity in the astrocyte during epileptogenesis. Acute hippocampal slices were prepared from Cre-positive, male and female GFAP-Cre; PC::G5-tdT mice. Calcium activity was reported by GCaMP5G expression. During 2-P imaging, aCSF, ATP ( $\mathrm{P}_{2} \mathrm{Y}$ agonist, 500 $\mu \mathrm{M}$ ), and DHPG (mGluR1/5 agonist, $500 \mu \mathrm{M}$ ) were focally applied near stratum radiatum astrocytes by an investigator blinded to solution identity.

We developed an unbiased approach to analyze astrocyte calcium responses to focal agonists, eliminating the need for userdefined ROIs. Briefly, we included an AlexaFluor-568 dye (30 $\mu \mathrm{M})$ in the agonist pipette and calculated the area of dye spread through simple ImageJ subtraction techniques (Fig. 2A). Because the dye (and presumably the agonist) spread across this area (mean \pm SEM area: $11,747 \pm 321 \mu \mathrm{m}^{2}$ ) over a $7-10 \mathrm{~s}$ period, calcium analyses $(\Delta \mathrm{F} / \mathrm{F}$ value $)$ are summated over a $10 \mathrm{~s}$ period $(\Delta \mathrm{F} / \mathrm{F} \cdot \mathrm{s}$ value $)$ to include later activation. Using this dye-spread ROI approach for analysis, we find that aCSF application alone does not induce any clear patterns of calcium activity within the dye-spread region over a $10 \mathrm{~s}$ period (Fig. $2 \mathrm{~B}, \mathrm{C}$ ). By contrast, ATP application consistently induces calcium activity within the dye-spread region (Fig. 2D). When results from the dye-spread ROI approach are compared with more traditional approaches, studying $\Delta \mathrm{F} / \mathrm{F}$ calcium activity in the soma of an astrocyte (Fig. $2 C$, blue traces for aCSF; Fig. $2 E$, orange traces for ATP), the same conclusions are apparent regarding astrocyte calcium activity in response to aCSF and ATP. However, for ATP application, the dye-spread ROI approach (black dotted line) does not fully capture the amplitude of $\Delta \mathrm{F} / \mathrm{F}$ calcium rises in the soma following ATP application, but it does track the overall kinetics of calcium activity. Overall, the dye-spread ROI approach strongly distinguishes the effects of aCSF versus ATP on astrocyte calcium activity within an unbiased region (main drug effect: $F_{(1,68)}=$ 57.38, $p<0.0001$; two-way ANOVA; $N=41$ animals in aCSF trials, $N=35$ animals in ATP trials; 1 slice/animal; Fig. $2 F$ ). Additionally, when aCSF and ATP calcium responses are studied across conditions (naive: $N=6$ animals, 1 slice/animal) and time points in KA-SE epileptogenesis $(24-72 \mathrm{~h}: N=8 ; 7-9 \mathrm{~d}: N=8$; 28-30 d: $N=11,1$ slice/animal), we did not uncover a main group effect (naive or KA-SE time period: $F_{(3,68)}=0.0754, p=$ 0.9730 ; two-way ANOVA; Fig. $2 F)$ or interaction effect $\left(F_{(3,68)}=\right.$ $0.9145, p=0.4387$; two-way ANOVA; Fig. $2 F$ ) for aCSF or ATP application on astrocyte calcium activity. These results suggest that (1) astrocyte calcium activity is not unduly influenced by pressure (aCSF application) at any stage of epileptogenesis; and 
A

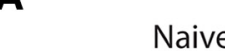

KA-SE: Acute

KA-SE: Acute

KA-SE: Latent

24-48 h

$72 \mathrm{~h}$ Day 7
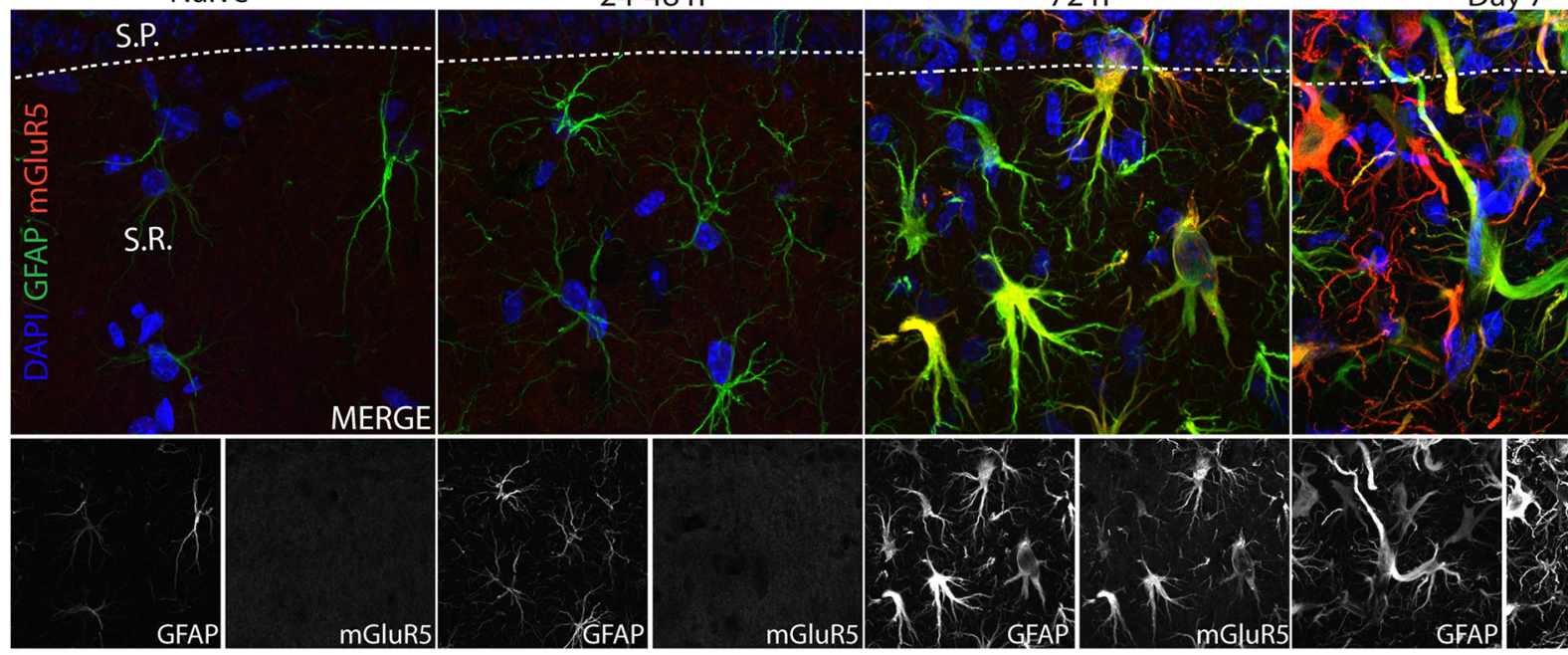

KA-SE: Chronic, Non-Epilepsy Day 28-35

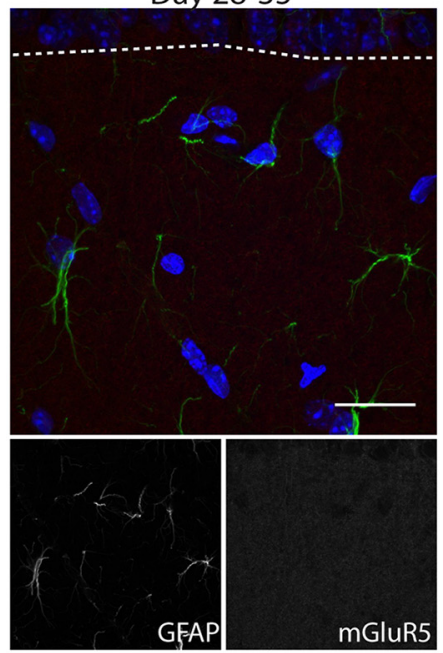

B

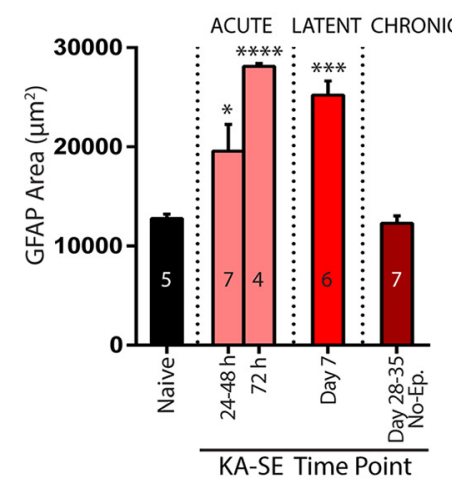

E
C

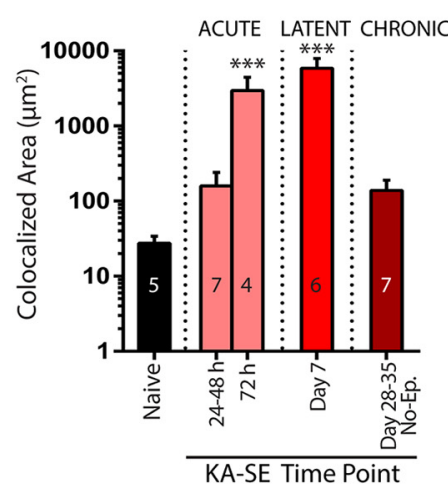

D

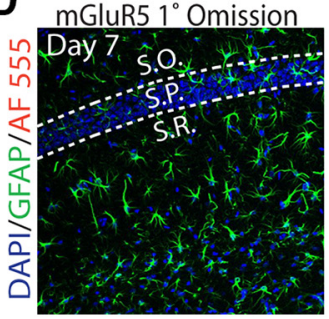

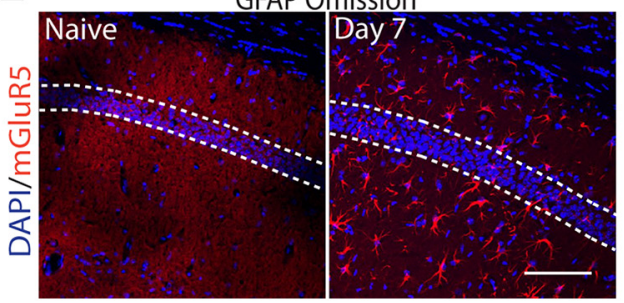

Figure 1. Astrocyte hypertrophy and mGluR5 expression in hippocampal stratum radiatum following KA-SE. A, Representative immunofluorescent micrographs of astrocytes (GFAP, bottom left panels), mGluR5 expression (bottom right panels), and nuclei (DAPI) in a merged image (main panel). Micrographs are of the stratum radiatum region of hippocampus, ventral to CA1 stratum pyramidale (white dashed line). Scale bars: main panels, $20 \mu \mathrm{m}$. B, Changes in GFAP area are quantified across time points in KA-SE pathology, depicted in $A$, to assess hypertrophy as a measure of reactive astrogliosis. C, Colocalized areas between mGluR5 and GFAP immunofluorescence are quantified across time points in KA-SE pathology, depicted in $\boldsymbol{A}$. B, C, Bar graphs represent mean \pm SEM (animal $N$ values provided in associated bar). Due to unequal variance, data values in $C$ were converted to $\log _{10}$ values, restoring equal variance, before performing statistical analyses. One-way ANOVA with Dunnett's post hoc test versus naive: ${ }^{*} p<0.05 ;{ }^{* * *} p<0.001$; ${ }^{* * *} p<0.0001$. $\boldsymbol{D}, \boldsymbol{E}$, The $20 \times$ magnification immunofluorescent micrographs of antibody omission. $\boldsymbol{D}$, Staining of DAPI, GFAP in the absence of mGluR5 primary antibody (secondary antibody: AlexaFluor-555). $E$, Staining of DAPI nuclei and mGluR5 in the absence of preconjugated GFAP-AlexaFluor-488 antibody. Patterns of mGluR5 staining appear to label cells with astrocyte-like morphology in day 7 KA-SE tissue (right), but not in naive tissue (left). Scale bars: D, E, 100 $\mu$ m. S.0., Stratum oriens; S.P., stratum pyramidale; S.R., stratum radiatum.

(2) ATP-induced calcium activity is reliably observed in astrocytes across all groups and time periods studied. Notably, ATP evokes calcium transients in the astrocyte through Gq-PLC-IP ${ }_{3}$ signaling, the canonical pathway of mGluR5 calcium signaling (Ferraguti and Shigemoto, 2006), indicating that Gq signaling could be initiated in the astrocyte across all conditions.

Using the dye-spread ROI approach for quantification, we next determined whether activation of mGlu5 receptors could induce calcium signaling in the astrocyte during epileptogenesis by focally applying the mGluR1/5 agonist DHPG (Fig. 3A). A 500 $\mu \mathrm{M}$ concentration of DHPG was chosen for these experiments because it more uniformly elicited calcium responses across our entire, unbiased ROI (Fig. 3A,B). Astrocyte calcium responses to DHPG reliably follow mGluR5 expression patterns (Fig. 1). For mature, naive astrocytes in stratum radiatum $(N=10$ animals, 1 slice/animal), focal application of DHPG does not produce levels of calcium activity in the astrocyte that are distinguishable from inert aCSF application $\left(F_{(6,66)}=25.22, p=0.9999\right.$; one-way ANOVA with Dunnett's post hoc comparison with aCSF aggregate trials; Fig. $3 C, D$ ). This suggests a lack of clear mGluR5 func- 
tion in the mature, naive astrocyte, consistent with our IHC data (Fig. 1) and reports of low mGluR5 transcript levels (Sun et al., 2013; Morel et al., 2014). We similarly observe that astrocytes studied 24-48 h after KA-SE $(N=5)$ do not display DHPG-induced calcium responses ( $p=0.9981$ vs aCSF, one-way ANOVA; Fig. $3 C, D)$. On the other hand, $72 \mathrm{~h}$ after KA-SE $(N=5)$, focal DHPG application could elicit a robust and prolonged calcium response in stratum radiatum astrocytes $(p<0.0001$ vs aCSF, one-way ANOVA; Fig. $3 C, D$ ), at a time point consistent with our first observations of mGluR5 colocalization with the astrocyte (Fig. 1). Similarly, in the latent period (day 7-9 after KA-SE, $N=8$ ), focal DHPG application consistently evoked calcium transients in stratum radiatum astrocytes $(p<0.0001$ vs aCSF, one-way ANOVA; Fig. $3 A, C, D$ ), suggesting that mGluR5 is functionally coupled to calcium signaling pathways in the astrocyte soon after KA-SE and into the seizure-free latent period.

In the chronic period (28-30 d after KA-SE), DHPG calcium responses are clearly distinct in acute slices prepared from animals with and without epilepsy development. We do not observe DHPGinduced calcium responses in stratum radiatum astrocytes studied in slices obtained from mice that did not develop epilepsy $(N=12,24 / 7$ video EEG monitoring; $p=0.9749$ vs aCSF; one-way ANOVA; Fig. $3 C, D$ ). These results are consistent with low to absent mGluR5 expression in fixed tissue prepared from nonepileptic KA-SE animals (day 28 or 35 after KA-SE; Fig. 1). In acute sections prepared from animals displaying documented, spontaneous seizures $(N=5$, 24/7 video EEG), stratum radiatum astrocytes consistently respond to DHPG application with increases in intracellular calcium ( $p<0.0001$ vs aCSF; one-way ANOVA; Fig. $3 C, D)$, suggesting that mGluR5 function is maintained into epilepsy. Interestingly, DHPG-induced calcium responses were observed across the epilepsy cohort regardless of whether a spontaneous seizure had occurred $4 \mathrm{~d}$ or up to $18 \mathrm{~d}$ before the date of acute slice preparation (day 28-30 after KA-SE). While we did not capture animals developing epilepsy 28-35 d after KA-SE in the IHC cohort, neuropathology studies consistently demonstrate that astrocytes in an epileptic network can express mGluR5, including hippocampal astrocytes in TLE tissue (Tang et al., 2001). Overall, focal agonist application studies demonstrate
A
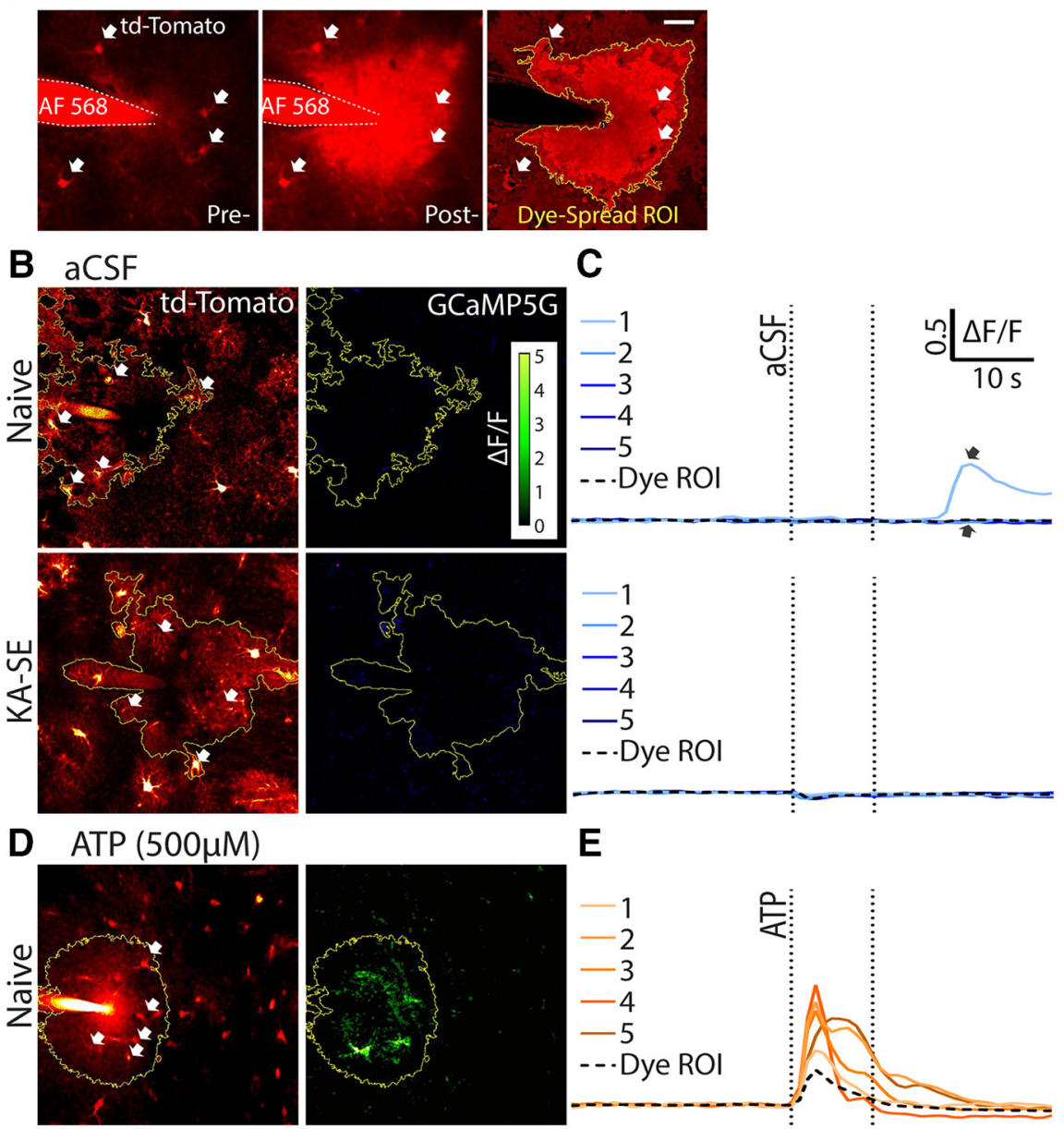

E
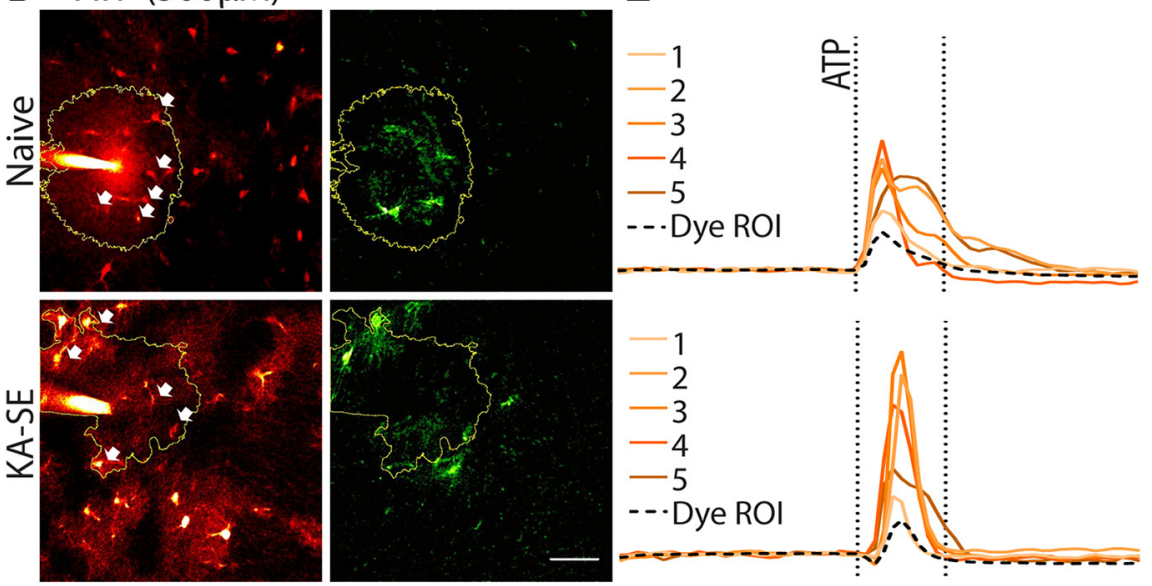

$\mathbf{F}$

Dye $\mathrm{ROI} \mathrm{Ca}^{2+}$ Activity

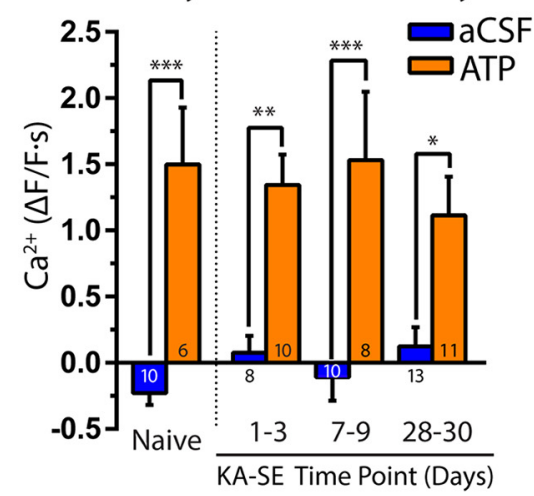

Figure 2. aCSF and ATP application yields consistent negative and positive calcium responses, respectively, using the dyespread ROI calcium analysis technique. A, 2-P micrographs showing td-Tomato-labeled astrocytes (white arrows) in stratum radiatum near a focal agonist pipette, filled with AlexaFluor-568 (AF 568) dye. By subtracting a maximum intensity projection of dye-spread over multiple frames (middle) from a single frame image captured before focal application (left), we can acquire an unbiased ROI to analyze calcium activity (dye-spread ROI, right). Scale bar, $25 \mu \mathrm{m}$. B, D, Left panels, 2-P micrographs of td-Tomatolabeled astrocytes (white arrows) within the overlaid area of dye-spread (yellow line) in representative naive and KA-SE trials. Right panels, GCaMP5G calcium fluorescence, depicted as the maximum intensity over a 10 s period following aCSF application(Figure legend continues.) 

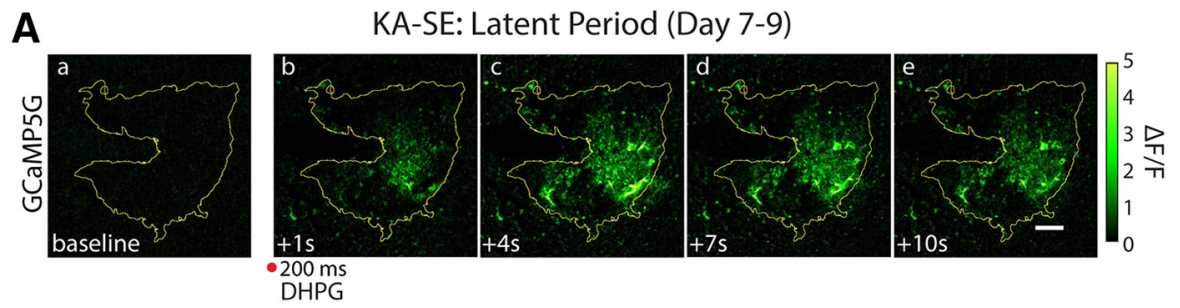

C
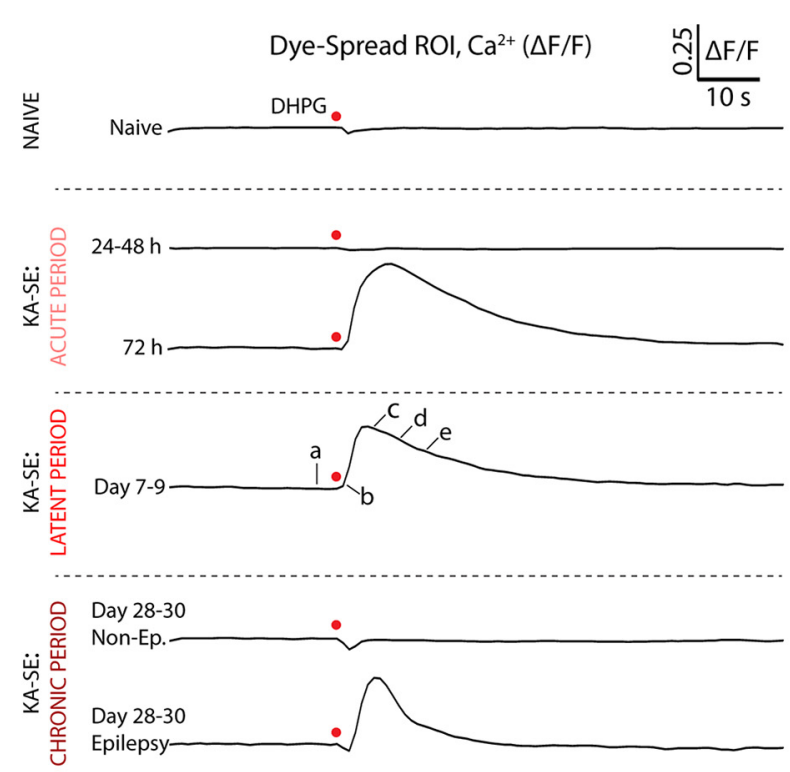
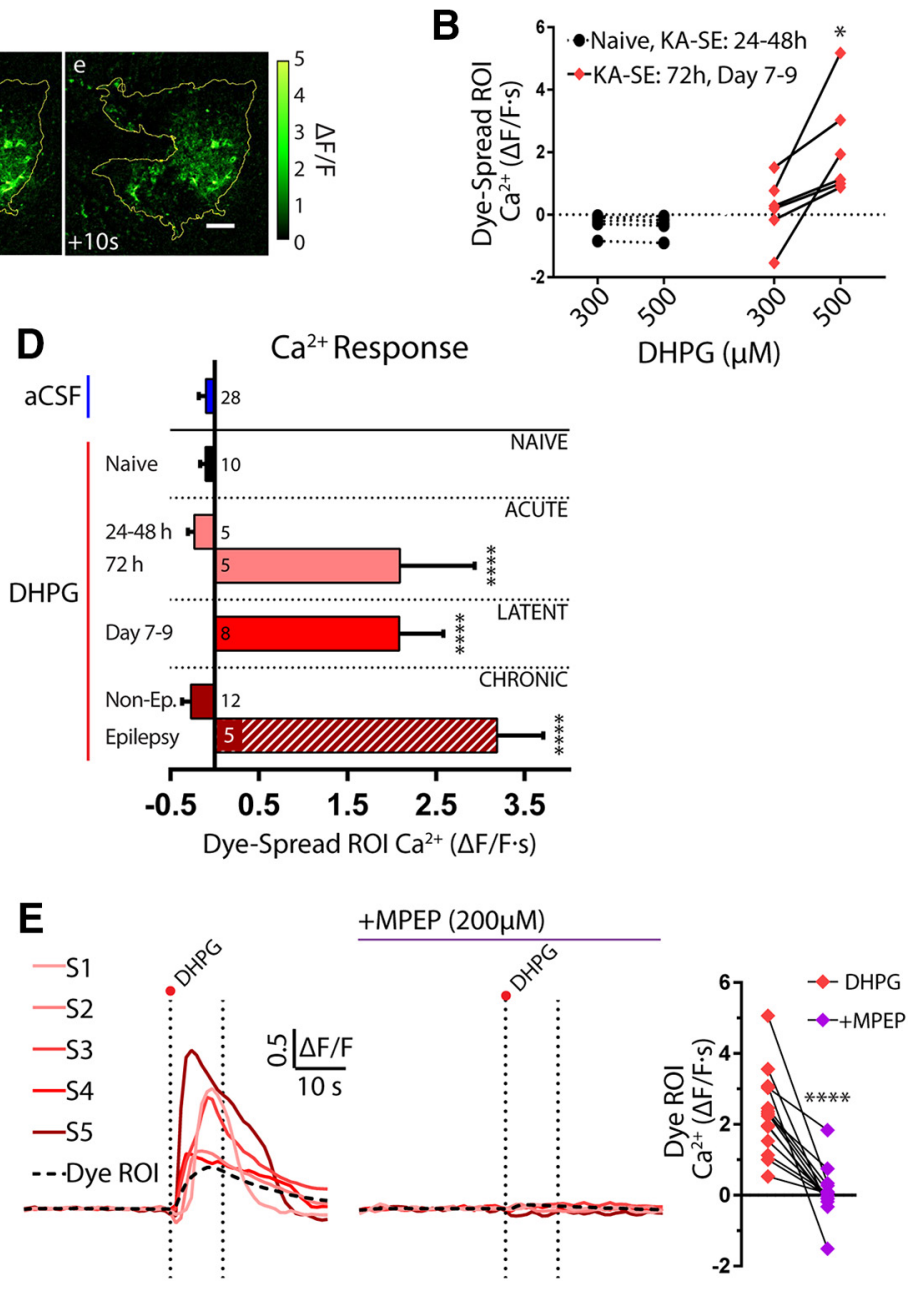

Figure 3. Time course of astrocyte mGluR5-mediated calcium activity in response to focal agonist application. $\boldsymbol{A}, \Delta \mathrm{F} / \mathrm{F}$ images of GCaMP5G fluorescence at baseline (Aa) and following DHPG application $(500 \mu \mathrm{m} ; \boldsymbol{A} \boldsymbol{b}-\boldsymbol{A e}$ ) in an acute hippocampal slice prepared in the latent period. Scale bar, $25 \mu \mathrm{m}$. $\boldsymbol{B}$, Focal DHPG application does not display a concentration-dependent effect on astrocyte calcium activity in naive slices or slices prepared $24-48 \mathrm{~h}$ after KA-SE (black circles) but does display a concentration-dependent effect on calcium activity in slices prepared $72 \mathrm{~h}$ or $7-9 \mathrm{~d}$ after KA-SE (red diamonds). Two-tailed, paired $t$ test: ${ }^{*} p<0.05$. C, Representative $\Delta \mathrm{F} / \mathrm{F}$ calcium responses within the dye-spread ROI when DHPG is focally applied to stratum radiatum astrocytes in the naive condition and at multiple time points in KA-SE epileptogenesis. In the latent period trace, lowercase letters (a-e) correspond to $\Delta \mathrm{F} / \mathrm{F}$ calcium images depicted in $\boldsymbol{A a}-\boldsymbol{A e}$, respectively. In the chronic period (28-30 d after KA-SE), responses are subdistinguished based upon whether the animal developed epilepsy by this period or not (Non-Ep.) using 24/7 video EEG. D, Bar graph represents the mean \pm SEM calcium response to DHPG application across the groups ( $N$ values provided in bar graph). One-way ANOVA with Dunnett's post hoc test versus aCSF (all aCSF trials, pooled): ${ }^{* * * *} p<$ 0.0001 . $E$, A representative paired trial in which a calcium response to DHPG was retested in the presence of the mGluR5-specific antagonist MPEP (200 $\mu \mathrm{M}$, bath application). Line graph represents paired calcium responses to DHPG in the absence (red diamonds) and presence (purple diamonds) of MPEP; $N=11$. Two-tailed, paired $t$ test: ${ }^{* * *} p<0.0001$ ).

that mGluR5 activation can induce calcium signaling in astrocytes across multiple periods in KA-SE epileptogenesis ( $72 \mathrm{~h}$, day 7-9) and in epilepsy. Furthermore, patterns of mGluR5 activation suggest that persistent mGluR5 expression/function in the astrocyte may be a reliable correlate of epileptogenesis, whereas

(Figure legend continued.) $\quad$ (B) or ATP application $(500 \mu \mathrm{m}, \boldsymbol{D})$. Scale bars: $\boldsymbol{B}, \boldsymbol{D}, 50 \mu \mathrm{m}$. C, Line graphs depicting $\triangle \mathrm{F} / \mathrm{F}$ calcium activity in the soma of astrocytes after aCSF application (corresponding to white arrows in $\boldsymbol{B}$ ) and within the entire dye-spread ROI (black dashed line). The dye-spread ROl is insensitive to a spontaneous event (black arrow) occurring in a single soma. $\boldsymbol{E}$, Line graphs representing $\Delta \mathrm{F} / \mathrm{F}$ calcium activity in the soma of astrocytes after ATP application (corresponding to white arrows in $\boldsymbol{D}$ ) and within the entire dye-spread ROI (black dashed line). $\boldsymbol{F}$, Bar graph represents mean \pm SEM calcium changes within the dye-spread ROl over a $10 \mathrm{~s}$ period $(\Delta \mathrm{F} / \mathrm{F} \cdot \mathrm{s})$ and compares the magnitude of aCSF calcium responses with ATP calcium responses across groups (naive and KA-SE time periods). The number of animals used for each agonist and group is displayed in the corresponding bar. Two-way ANOVA with Sidak's post hoc test: ${ }^{*} p<0.05$; ${ }^{* *} p<0.01 ;{ }^{* * *} p<0.001$. transient mGluR5 expression/function may signify the resolution of an injury response.

DHPG calcium responses are specific to mGluR5 activation and are not influenced by endocannabinoid signaling In a subset of trials in which DHPG application led to a clear calcium response in the astrocyte (at 72 h, day 7-9, or in epilepsy), we investigated the specificity of the calcium response to mGluR5 activation. DHPG-induced calcium activity did not persist when mGluR5 signaling was blocked by bath application of the specific antagonist MPEP $\left(200 \mu \mathrm{M} ; t_{(13)}=6.288, p<0.0001\right.$; paired $t$ test; Fig. $\left.3 E\right)$. While all agonist studies (Figs. 2, 3) were performed in the presence of TTX (500 nM), action-potential-independent release of endocannabinoids could also induce calcium activity in the astrocyte (Fig. 4). As depicted in Figure $4 A$, we tested an alternative possibility that DHPG induced postsynaptic endocannabinoid release (Tabatadze et al., 2015; Colavita et al., 2016; Marshall et al., 2018), evoking calcium activity in the astrocyte through noncanonical CB1 receptor 
A

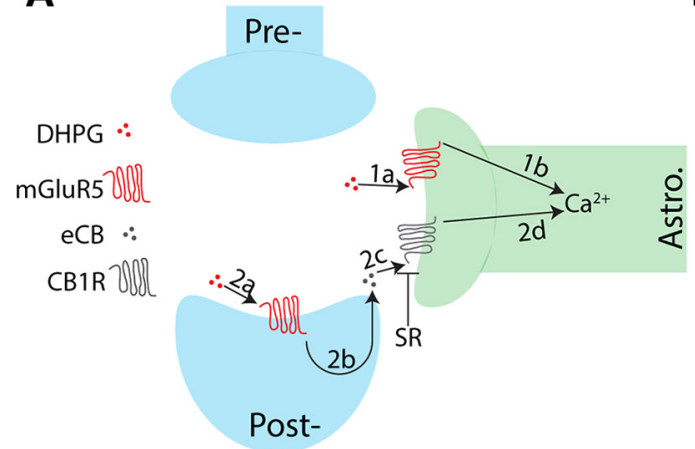

B

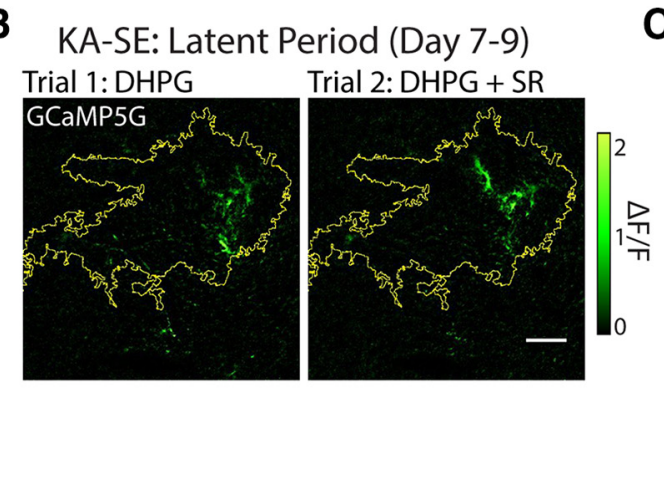

C

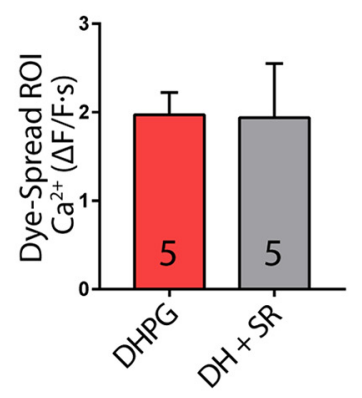

Figure 4. Assessing potential endocannabinoid-mediated calcium activity downstream of DHPG application. $\boldsymbol{A}$, Model for potential confounding effects of DHPG application at the astrocyteneuron tripartite synapse. Model 1, Focal DHPG application may directly activate mGluR5, expressed on the astrocyte (1a), leading to calcium activity in the astrocyte (1b) through canonical intracellular signaling cascades. Model 2, Focal DHPG application may activate postsynaptic mGluR1/5 (2a), leading to retrograde endocannabinoid release (2b), which would not be blocked by TTX $(500 \mathrm{~nm})$ in the bath. Endocannabinoids may then activate cannabinoid 1 receptors (CB1R, 2c), expressed by the astrocyte. (B1R signaling could then activate calcium activity in astrocytes through noncanonical intracellular calcium signaling pathways (2d). If calcium responses to DHPG were mediated through a Model 2 mechanism, bath application of the selective CB1R antagonist SR-141716A (SR) is predicted to attenuate calcium activity in the astrocyte. Experiments were conducted in acute slices ( $N=5$ animals) prepared 7-9 d after KA-SE. $B$, Micrographs depicting GCaMP5G calcium activity in astrocytes following focal application of DHPG (500 $\mu \mathrm{m}$ ). Trial 1, Baseline DHPG responses in bath aCSF (left). Trial 2, DHPG responses in the presence of bath SR-141716A (10 $\mu \mathrm{M}$, right). Scale bar, $25 \mu \mathrm{m}$. C, Bar graph represents mean \pm SEM calcium activity within the dye-spread R0I during DHPG application alone or in combination with bath SR-141716A. Calcium activity is not attenuated by SR-141716A application.

signaling mechanisms (Lauckner et al., 2005; Bonilla-Del Riotao et al., 2017). In our hands, we do not find that the blockade of CB1R signaling mechanisms (SR-141716A, CB1R-specific antagonist, 10 $\mu \mathrm{M})$ alters DHPG-induced calcium activity in the astrocyte in a cohort of latent period animals $\left(N=5\right.$, day 7-9 after KA-SE; $t_{(4)}=$ $0.0682, p=0.9489$; paired $t$ test; Fig. $4 B, C$ ). In conclusion, antagonist studies establish the mGluR5 specificity of DHPG calcium responses and suggest that observations of calcium activity are not confounded by TTX-insensitive endocannabinoid signaling.

\section{Generating an astrocyte-specific, mGluR5 conditional knock-out line}

After determining that hippocampal astrocytes can express functional mGlu5 receptors in the context of epileptogenesis, we developed and characterized a genetic approach to study the loss of astrocyte mGluR5 signaling during epilepsy development. We bred an mGluR5 ${ }^{\mathrm{f} / \mathrm{fl}}$ line to an astrocyte-specific Cre line (GFAP$\mathrm{CreER}^{\mathrm{T} 2}$ or ALDH1L1-CreER ${ }^{\mathrm{T} 2}$ ) and a fluorescent reporter/calcium indicator line (PC::G5-tdT). Tamoxifen-induced recombination (at 6-9 weeks of age) in our line (mGluR5 ${ }^{\mathrm{fl} / \mathrm{fl}}$; PC::G5-tdT ${ }^{\text {het }}$; ALDH1L1-CreER ${ }^{\mathrm{T} 2}$ or GFAP-CreER ${ }^{\mathrm{T} 2}$, referred to as $\mathrm{mGluR}^{-1-}$ ) should only prohibit mGluR5 expression in astrocytes after early development. Additionally, td-Tomato expression should identify astrocytes that underwent recombination in slice and allow us to study calcium activity in those astrocytes (GCaMP5G). In our studies, mGluR5 ${ }^{+/+}$mice (mGluR5 ${ }^{\mathrm{w} / \mathrm{w}}$; PC::G5-tdT ${ }^{\text {het }}$; ALDH1L1$\mathrm{CreER}^{\mathrm{T} 2}$ or GFAP-CreER ${ }^{\mathrm{T} 2}$ ), derived from a parallel breeding scheme, were used as a control line.

To characterize the mGluR5 ${ }^{+/+}$and GGluR5 $^{-/-}$backgrounds, we investigated calcium responses to focal DHPG application (500 $\mu \mathrm{M}$ ) in slice, 7-9 d after KA-SE (KA-SE:mGluR5 ${ }^{+/+}, N=4,1-2$ slices/animal; KA-SE:mGluR5 ${ }^{-1-}, N=7,1-2$ slices/animal), and compared responses to naive slices prepared from both genetic backgrounds ( $N=8$ total; $N=4$ of each genotype, $1-2$ slices/animal). Across all experiments using mGluR5 ${ }^{+/+}$and mGluR5 ${ }^{-/-}$mice (see Figs. 5, 6, 7, 8), we did not detect behavioral differences in KA-SE seizure severity between these lines (cumulative seizure burden during KA-SE: $47 \pm 16 \mathrm{mGluR}^{+/+}$vs $40 \pm 9$ mGluR5 $5^{-/-} ; p=0.2103$, Student's $t$ test). We found that only $3 \%$ of naive astrocytes surveyed ( $n=2$ of 61 cells of either an mGluR $5^{+/+}$or $\mathrm{mGluR} 5^{-/-}$genotype) demonstrated a reproducible calcium response to DHPG application (Fig. 5). Similar to previous findings (Fig. 3), the vast majority $(92 \%)$ of td-Tomato-labeled astrocytes in KA-SE:mGluR5 ${ }^{+/+}$tissue displayed DHPG-induced calcium responses $(n=92$ of 100 cells; Fig. 5). By comparison, $82 \%$ of KA-SE:mGluR5 ${ }^{-1-}$ astrocytes surveyed did not exhibit a reproducible DHPG response $(n=79$ of 96 cells; Fig. 5). These results suggest that, after recombination (tdTomato labeling), the majority of $\mathrm{mGluR} 5^{-1-}$ astrocytes lose mGluR5-dependent calcium signaling in the latent period.

\section{Following KA-SE, synaptic glutamate increases astrocyte calcium activity through mGluR5 signaling}

We next used mGluR5 ${ }^{+/+}$and mGluR5 ${ }^{-1-}$ animals to investigate how astrocyte-neuron synaptic communication is influenced by the presence or absence of astrocyte mGluR5 signaling. In early development, astrocyte mGluR5 activation can induce multiple forms of astrocyte-neuron interactions (both structural and functional) in response to high-frequency neuronal activity (Devaraju et al., 2013; Bernardinelli et al., 2014). We tested whether astrocyte mGluR5 signaling can be activated by highfrequency neuronal activity $(40 \mathrm{~Hz}, 1$ s stimulation of the Schaffer collateral pathway; Fig. 6A) during epileptogenesis (Szokol et al., 2015). To do so, we pharmacologically isolated mGluR5dependent calcium activity from overall astrocyte calcium activity using the mGluR5-specific antagonist MPEP $(75 \mu \mathrm{M})$. In our study, fEPSP amplitudes are consistent across groups and bath conditions (aCSF alone or aCSF + MPEP) during the high-frequency stimulus $\left(F_{(5,20)}=0.4581, p=0.8026\right.$; oneway ANOVA; Fig. $6 C$ ), and in singly evoked fEPSPs studied between trials $\left(t_{(13)}=0.7817, p=0.4484\right.$; paired $t$ test; Fig. $6 B)$. This suggests that levels of presynaptic neurotransmitter release are not significantly altered by prior high-frequency stimulation, or the presence of bath MPEP application.

We first determined whether overall astrocyte calcium activity following afferent stimulation differed between groups (Fig. 6D). In response to $40 \mathrm{~Hz}$ neuronal stimulation, KA-SE:mGluR5 ${ }^{+/+}$ astrocytes $(N=5$ animals $)$ displayed significantly larger calcium transients $(\Delta \mathrm{F} / \mathrm{F} \cdot \mathrm{s})$ in response to neuronal stimulation than KA-SE:mGluR5 ${ }^{-/-}$astrocytes $\left(N=5\right.$ animals; $F_{(2,10)}=5.226$, $p=0.0279$; one-way ANOVA with Tukey's post hoc test; Fig. 
A
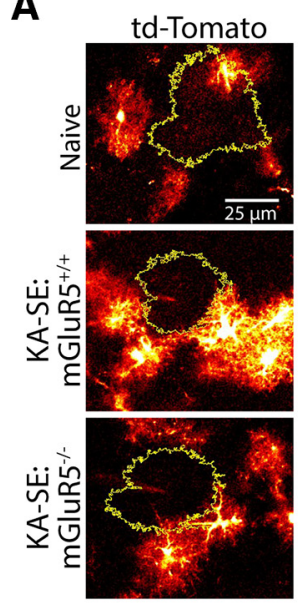

B
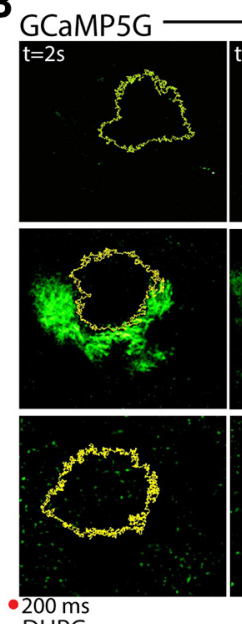

DHPG
C

\section{Astrocytes Responding to $500 \mu \mathrm{M}$ DHPG}

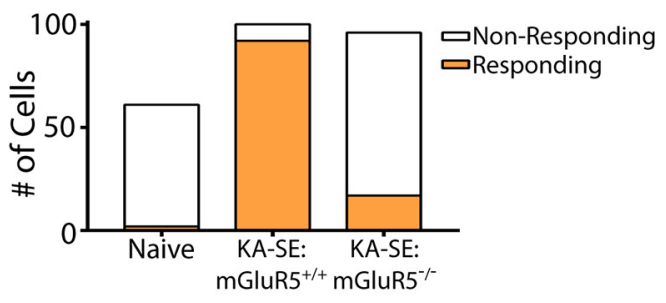

Figure 5. Astrocyte-specific mGluR5 knock-out reduces the proportion of astrocytes with mGluR5-mediated calcium responses following KA-SE. A, td-Tomato-labeled astrocytes and the dye-spread area of focal DHPG application (500 $\mu$ m, yellow line). B, GCaMP5G calcium activity $2 \mathrm{~s}$ (left panels), $4 \mathrm{~s}$ (middle panels), and $6 \mathrm{~s}$ (right panels) after focal DHPG application. Scale bars: $A$, $B, 25 \mu \mathrm{m}$. C, Bar graphs displaying the number of astrocytes within $40 \mu \mathrm{m}$ of the dye-spread ROl that responded to focal DHPG application ( $>0.5 \Delta \mathrm{F} / \mathrm{F}$ calcium change in the soma or a major branch). In naive tissue, $n=2$ of 61 astrocytes surveyed in stratum radiatum displayed a calcium response to focal DHPG application (tissue from $N=8$ animals). In KA-SE:mGluR5 ${ }^{+/+}$tissue, $7-9$ $\mathrm{d}$ after KA-SE, $n=92$ of 100 astrocytes surveyed in stratum radiatum displayed a calcium response to focal DHPG application (tissue from $N=5$ animals). In KA-SE:mGluR5 ${ }^{-/}$tissue, $7-9 \mathrm{~d}$ after KA-SE, $n=17$ of 96 astrocytes surveyed in stratum radiatum displayed a calcium response to focal DHPG application (tissue from $N=7$ animals).

$6 D, F)$. Some calcium activity is expected across all groups because astrocytes express $\mathrm{P}_{2} \mathrm{Y}$ receptors (Dixon et al., 2004), and consistently respond to neuronal or exogenous ATP release (Fig. 2) with intracellular calcium signaling (Porter and McCarthy, 1995; Bowser and Khakh, 2004; Tang et al., 2015). To test whether increased calcium responses in the latent period were specifically due to mGluR5-dependent calcium signaling, we bath-applied the mGluR5 antagonist MPEP $(75 \mu \mathrm{M})$. In naive tissue $(N=3$ animals), bath application of MPEP did not alter stimulationinduced astrocyte calcium activity $\left(t_{(2)}=1.078, p=0.3937\right.$; paired $t$ test; Fig. $3 D-F$ ). This would suggest that mGluR5 signaling does not clearly contribute to stimulation-induced calcium activity in the naive astrocyte, consistent with earlier studies (Figs. 1, 3, 5). On the other hand, mGluR5 ${ }^{+/+}$astrocytes in the latent period displayed a $51 \%$ attenuation in their calcium activity when MPEP was bath-applied during neuronal stimulation $\left(t_{(4)}=6.139, p=0.0035\right.$; paired $t$ test; Fig. $\left.6 D-F\right)$, suggesting that mGluR5-dependent calcium signaling can be triggered by neuronal glutamate release during epileptogenesis. Importantly, we did not observe that MPEP bath application affected calcium activity in KA-SE:mGluR5 ${ }^{-1-}$ astrocytes $\left(t_{(4)}=0.5034, p=0.6411\right.$; paired $t$ test; Fig. $6 D-F$ ), consistent with previous experiments (Fig. 5). This observation suggests that mGluR5 signaling does not substantially contribute to stimulation-induced calcium activity in the knock-out astrocyte following KA-SE. Overall, our stimulation-evoked calcium studies suggest that synaptically induced, mGluR5-dependent calcium signaling, an important component of astrocyte-neuron interaction in early development, can reemerge in KA-SE:mGluR $5^{+/+}$astrocytes but is either developmentally restricted in adult, naive astrocytes (Sun et al., 2013) or experimentally restricted in KA-SE: mGluR5 ${ }^{-/-}$astrocytes.

After KA-SE, astrocytes display faster glutamate uptake during low-frequency stimulation

To assess whether mGluR5 signaling in epileptogenesis influences astrocyte membrane properties and glutamate transport, we conducted whole-cell recordings in mGluR $5^{+/+}$and
mGluR5 ${ }^{-/-}$slices following KA-SE. Acute hippocampal slices were prepared 7-9 d after KA-SE. We used focal DHPG application to correctly identify and record from astrocytes without mGluR5 signaling in the knock-out condition (KA-SE:mGluR5 ${ }^{-1-}$; $n=18$ cells from $N=6$ animals), because the expression of td-Tomato in slice does not definitively mark astrocytes without mGluR5 signaling in the knock-out (Fig. 5). Similarly, focal DHPG application was used to include only astrocytes with mGluR5 signaling after KA-SE in the WT condition (KA-SE: mGluR5 ${ }^{+/+} ; n=15$ cells from $N=5$ animals). In the naive group (composed of both genotypes), astrocytes without mGluR5 signaling were confirmed for use in these studies $(n=15$ cells from $N=8$ animals). Verification of mGluR5 expression status in this fashion allowed us to definitively determine whether recombination events were inclusive of mGluR 5 in the knock-out line and also allowed us to avoid the rare astrocyte that might express mGluR5 in the naive state. We did not detect any differences in cell-intrinsic properties or recording properties between astrocytes with and without mGluR5 signaling after status epilepticus (KA-SE:mGluR5 ${ }^{+/+}$and KA-SE:mGluR5 ${ }^{-/-}$). However, both KA-SE:mGluR5 ${ }^{+/+}$and KA-SE:mGluR5 ${ }^{-1-}$ astrocytes displayed a modest lower access resistance value relative to naive astrocytes (naive, $10.84 \pm 0.32 \mathrm{M} \Omega$; KA-SE:mGluR5 ${ }^{+/+}$, $9.231 \pm$ $0.44 \mathrm{M} \Omega$; KA-SE:mGluR5 ${ }^{-1-}, 8.86 \pm 0.38 \mathrm{M} \Omega$; naive vs KA-SE: mGluR5 ${ }^{+/+}, p=0.0177$; naive vs KA-SE:mGluR5 ${ }^{-/-}, p=$ 0.0019; one-way ANOVA with Tukey's post hoc test).

We then evaluated glutamate transporter function in these astrocytes. GTCs were pharmacologically isolated from wholecell recordings following stimulation of the Schaffer collateral pathway (Bergles and Jahr, 1997). In initial trials, we studied the kinetics and characteristics of GTCs in response to single-pulse stimulations $(0.2 \mathrm{~Hz})$ at increasing stimulus intensities $(250 \mu \mathrm{A}$, $500 \mu \mathrm{A}, 1 \mathrm{~mA}, 3 \mathrm{~mA}$, and $6 \mathrm{~mA}$ ), intended to saturate GTC amplitude (Fig. 7A). At saturating stimulus intensities, the amplitude of the GTC was similar between groups $\left(F_{(2,45)}=1.912\right.$, $p=0.1597$, one-way ANOVA). Additionally, paired-pulse stimulation (3 mA, $40 \mathrm{~ms}$ interstimulus interval) did not indicate differences in facilitation between the three conditions (Fig. 7D). 
A

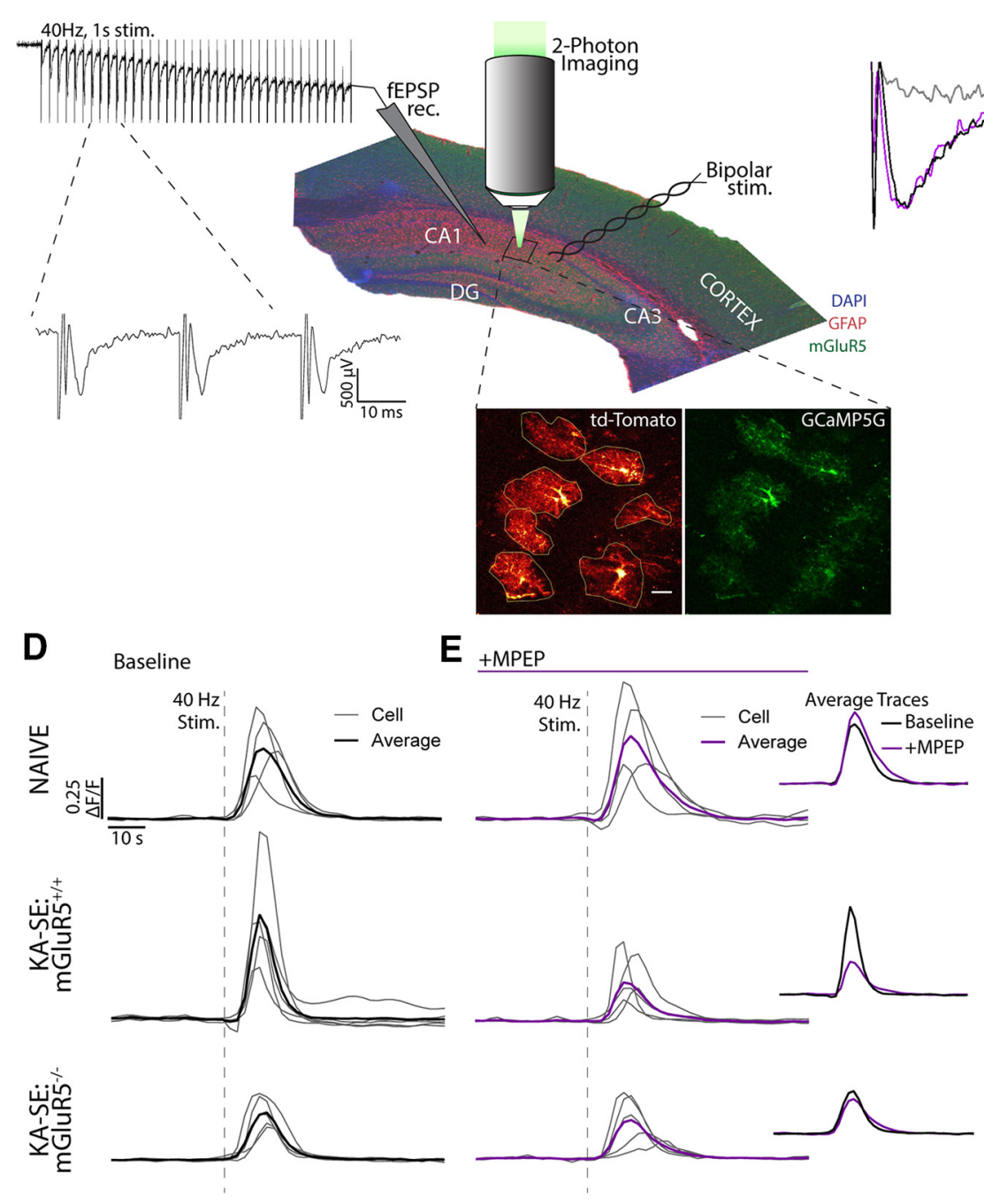

fEPSP $\quad 100 \mu \mathrm{v} \bigsqcup_{20 \mathrm{~ms}}$ -Baseline (aCSF) - MPEP - TTX

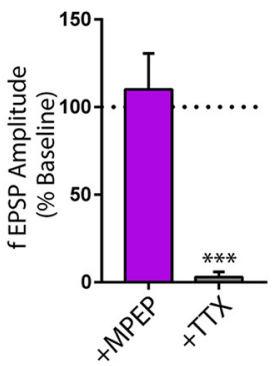

C fEPSP Response
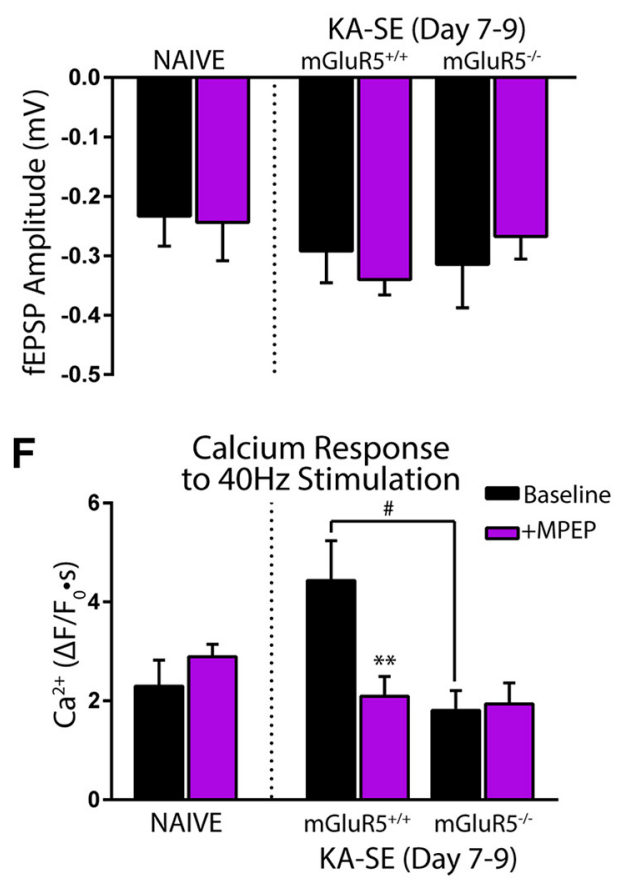

Figure 6. Astrocytes with mGluR5 expression in epileptogenesis exhibit calcium responses to high-frequency synaptic glutamate release. A, Depiction of the experimental setup. Bipolar stimulation of the Schaffer collateral pathway was used to induce local calcium activity in stratum radiatum astrocytes following neurotransmitter release. Calcium activity was imaged using 2-P microscopy. Yellow outlines indicate whole astrocyte ROI. Scale bar, $15 \mu \mathrm{m}$. fEPSPs and afferent stimulation $(40 \mathrm{~Hz}, 1 \mathrm{~s})$ were recorded by a local field recording electrode. $\boldsymbol{B}$, Representative traces depicting fEPSP recordings before a first $40 \mathrm{~Hz}$ stimulation ("Baseline") and before a second $40 \mathrm{~Hz}$ stimulation in the presence of MPEP (75 $\mu \mathrm{M}$ ). Abolition of the fEPSP recording in the presence of tetrodotoxin (TTX, $1 \mu \mathrm{M}$ ) is also shown. The corresponding bar graph represents the baseline-normalized mean \pm SEM fEPSP amplitude when MPEP is bath-applied (pooled data across all trials, $N=$ 13; two-tailed, paired $t$ test, $p=0.4484$ ) and when TTX is bath-applied (select trials, $N=2$ ). Two-tailed, Welch's $t$ test: ${ }^{* *} p=0.0001$. C, Bar graph displaying group mean \pm SEM fEPSP responses during $40 \mathrm{~Hz}$ stimulation across groups and bath conditions. No significant differences in fEPSP amplitude are detected between groups or conditions (one-way ANOVA). $D, \triangle F / F$ traces of baseline calcium activity in response to a $40 \mathrm{~Hz}$ stimulus (dotted line) for single astrocytes (gray traces). $\boldsymbol{E}$, Corresponding $\Delta \mathrm{F} / \mathrm{F}$ traces of calcium activity in the presence of MPEP when a $40 \mathrm{~Hz}$ stimulus (dotted line) is reapplied and studied in the same single astrocytes (gray traces). An overlay of average calcium responses in the representative baseline trials (black lines in $C$ ) and MPEP trials (purple lines in D). $\boldsymbol{F}$, Bar graph represents the group mean \pm SEM calcium activity in astrocytes when a $40 \mathrm{~Hz}$ stimulus is applied in the absence ("Baseline") or presence (" + MPEP") of the mGluR5 antagonist $\operatorname{MPEP}\left(N=3\right.$ naive, $N=5 \mathrm{KA}$-SE:mGluR5 ${ }^{+/+}$, and $N=5 \mathrm{KA}$-SE:mGluR5 $\left.{ }^{-1-}\right)$. Comparisons of the initial calcium response between groups used a one-way ANOVA design with Tukey's post hoc testing: ${ }^{\#} p<0.05$. Assessment of MPEP-induced changes in calcium activity used a within-subject design. Two-tailed, paired $t$ test, for KA-SE:mGluR5 ${ }^{+/+}$astrocytes: ${ }^{* *} p<0.01$.

Together, these findings suggest that presynaptic glutamate release is similar between naive tissue and KA-SE tissue, when studied using single or paired pulses.

While GTC amplitude was similar between groups, we noted significant differences in current decay kinetics between groups (Fig. 7C). GTCs recorded from stratum radiatum astrocytes after KA-SE (both KA-SE:mGluR5 ${ }^{+/+}$and KA-SE:mGluR5 ${ }^{-/-}$) exhibited faster first-order decay constants than naive astrocytes (first-order, exponential decay $\tau: F_{(2,45)}=15.2$; vs KA-SE: mGluR5 $^{+/+}, p<0.0001$; vs KA-SE:mGluR5 ${ }^{-/-}, p=0.0001$; one-way ANOVA with Tukey's post hoc test; Fig. 7C). Faster exponential decay constants following KA-SE reduced the overall GT current half-width, relative to naive astrocyte recordings
$\left(F_{(2,45)}=5.554\right.$; vs KA-SE:mGluR5 ${ }^{+/+}, p=0.0096$; vs KA-SE: mGluR5 ${ }^{-1-}, p=0.0287$; one-way ANOVA with Tukey's post hoc test; Fig. 7C). This suggests that KA-SE generally enhances the kinetics of astrocyte glutamate uptake during low-frequency stimulation, and is consistent with observations made in the rat KA-SE model (Takahashi et al., 2010). However, during lowfrequency stimulation, the faster kinetics of glutamate uptake after KA-SE do not appear to be mGluR5-dependent.

In a subset of trials, we also evaluated the specificity of the recorded current to glutamate transport. Following bath application of (3S)-3-[[3-[[4-(trifluoromethyl)benzoyl] amino]phenyl] methoxy]-L-aspartic acid (TFB-TBOA, $300 \mathrm{~nm}$ ), the high-affinity antagonist of astrocyte glutamate transporters (GLAST and GLT- 


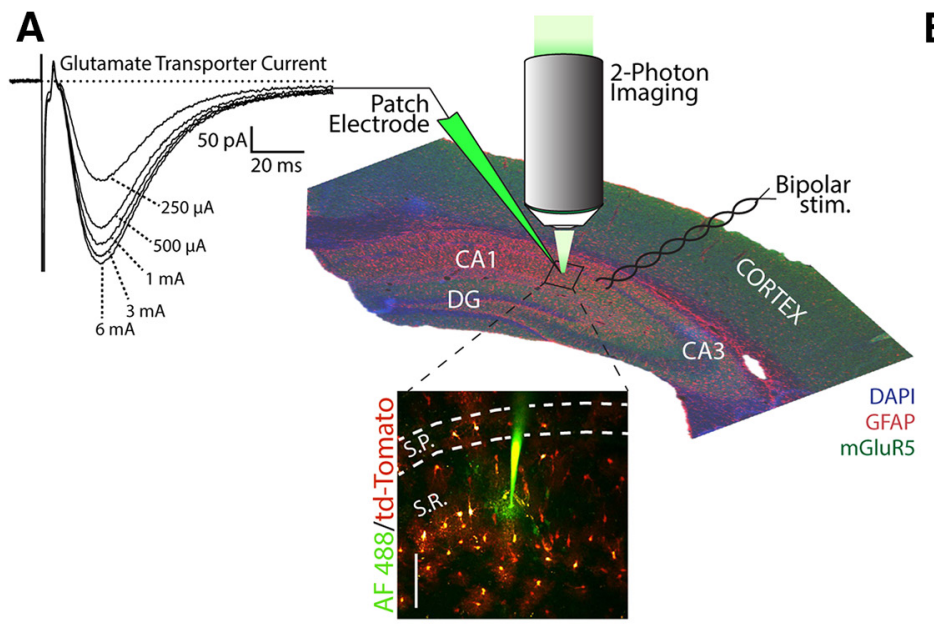

B

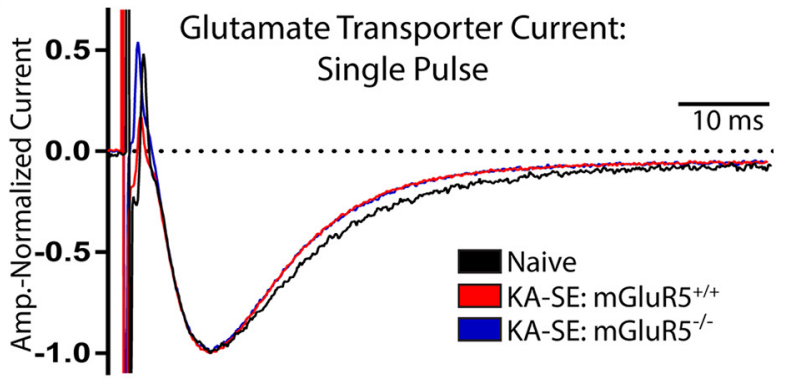

C
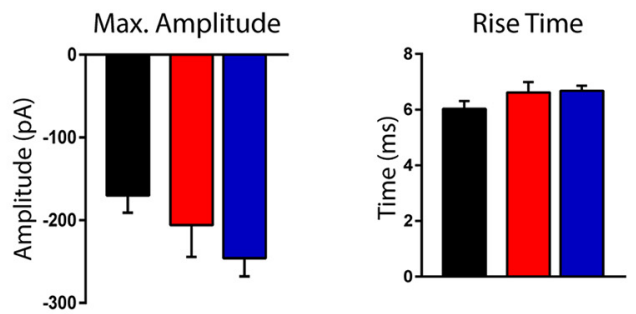
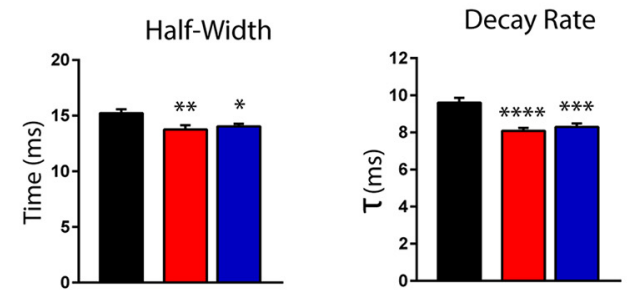

D

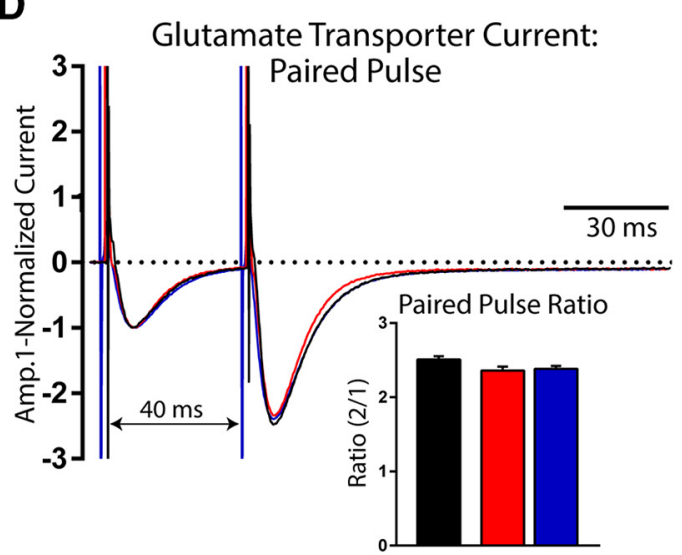

$\mathbf{E}$

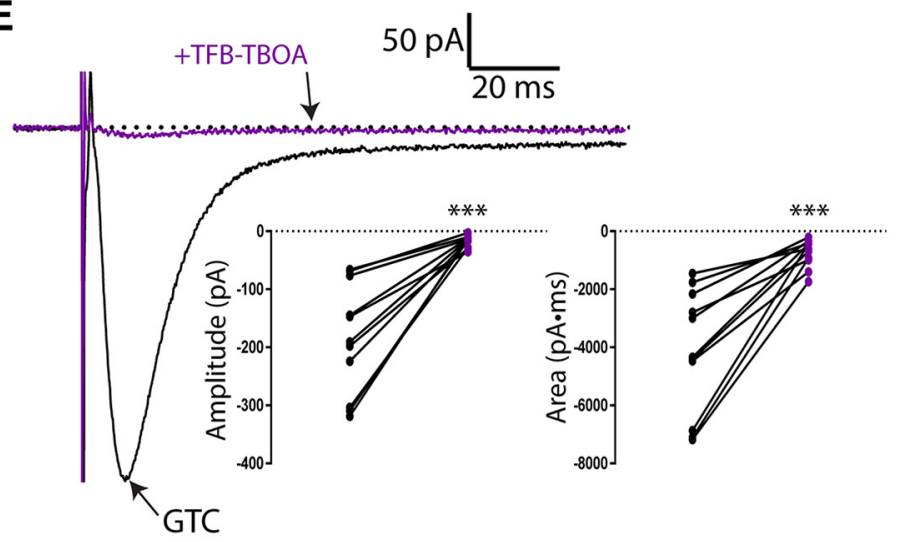

Figure 7. Synaptic glutamate uptake following low-frequency stimulation occurs more rapidly in the KA-SE latent period but is not influenced by mGluR5 signaling. $A$, GTCs in response to bipolar stimulation of the Schaffer collateral pathway at increasing stimulus intensities. $\boldsymbol{B}$, A representative GTC from a naive, KA-SE:mGluR5 ${ }^{+/+}$, and KA-SE:mGluR5 ${ }^{-/-}$astrocyte was peak-normalized to compare transporter current kinetics. C, Bar graphs represent mean \pm SEM values for the maximum amplitude, $0 \%-100 \%$ rise time, half-width, and single-exponential tau ( $\tau$ ) decay rate of the GTC recorded in response to single-pulse $(0.2 \mathrm{~Hz})$, afferent glutamate release. $D$, A representative GTC response to paired-pulse stimuli $(3 \mathrm{~mA}, 40 \mathrm{~ms}$ interval) was normalized to the first peak amplitude to compare paired-pulse facilitation between groups. The mean \pm SEM paired-pulse facilitation ratio for the GTC is depicted in the corresponding bar graph. $C, D, B a r$ graphs: $n=15$ cells from 8 animals in the naive group, $n=15$ cells from 5 animals in the KA-SE:mGluR5 ${ }^{+/+}$group, and $n=18$ cells from 6 animals in the KA-SE:mGluR5 ${ }^{-1-}$ group. One-way ANOVA with Tukey's posthoctest: ${ }^{*} p<0.05 ;{ }^{* *} p<0.01 ;{ }^{* * *} p<0.001 ;{ }^{* * *} p<0.0001$. E, A GTC in the absence (black trace) and presence of TFB-TBOA (300 nm, purple trace), the high-affinity antagonist of astrocyte glutamate transporters GLT-1 (EAAT2) and GLAST (EAAT1). The individual mean GTC amplitude and current area are shown for paired trials in the absence and presence of TFB-TBOA bath application. Two-tailed, paired $t$ test, $n=11$ astrocytes: ${ }^{* * *} p<0.001$.

1), we observed a strong attenuation of current amplitude $(-186.2 \pm 28.85 \mathrm{pA}$ aCSF vs $-17.19 \pm 28.19 \mathrm{pA}$ in aCSF with TFB-TBOA; $t_{(10)}=5.995, p=0.0001$; two-tailed, paired $t$ test; Fig. $7 E)$ and current area $(-4141 \pm 644 \mathrm{pA} \cdot \mathrm{ms}$ aCSF vs $-747 \pm$ $144 \mathrm{pA} \cdot \mathrm{ms}$ in aCSF with TFB-TBOA; $t_{(10)}=5.813, p=0.0002$; two-tailed, paired $t$ test; Fig. $7 E$ ).

Astrocytes with mGluR5 signaling in the latent period display enhanced glutamate uptake kinetics during high-frequency stimulation

We next assessed astrocyte glutamate uptake in response to high-frequency afferent stimulation (Fig. 8). High-frequency afferent stimulation can be used to assess glutamate clearance after summated activity, which can overwhelm transport capacity and produce extrasynaptic glutamate spillover if the kinetics of glutamate transport are altered (Diamond and Jahr, 2000; Tanaka et al., 2013). Additionally, high-frequency stimulation is more reflective of network activity during seizure. Ten stimuli were applied to the Schaffer collateral pathway in a high-frequency train $(100 \mathrm{~Hz}, 100 \mathrm{~ms})$ at a reliably saturating stimulus intensity ( $6 \mathrm{~mA}$; Fig. $8 \mathrm{~A}$ ). During the high-frequency train, naive astrocytes showed a marked increase in facilitation relative to the KA-SE astrocyte recordings (both KA-SE: 

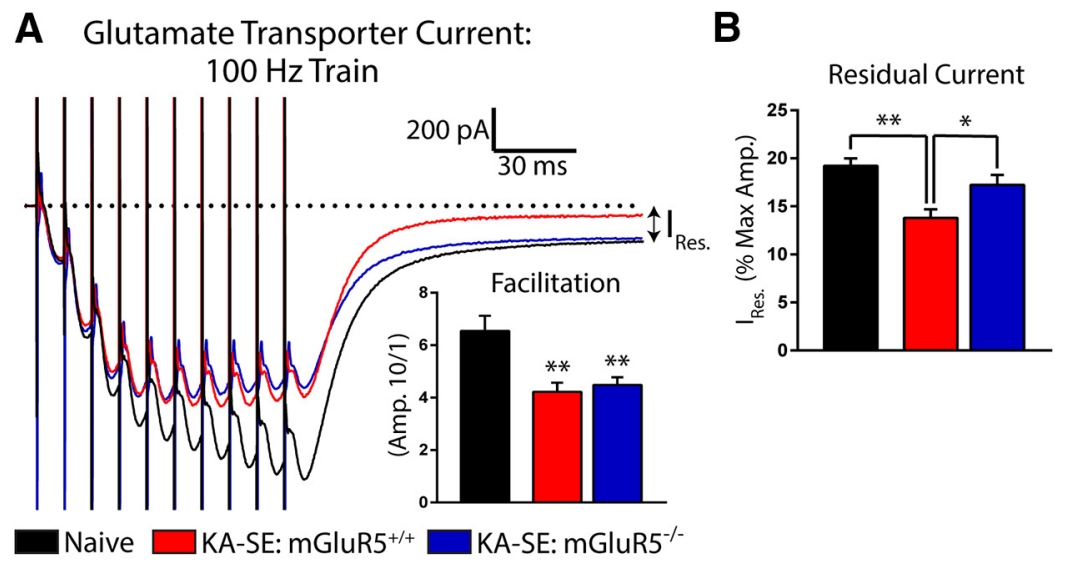

C

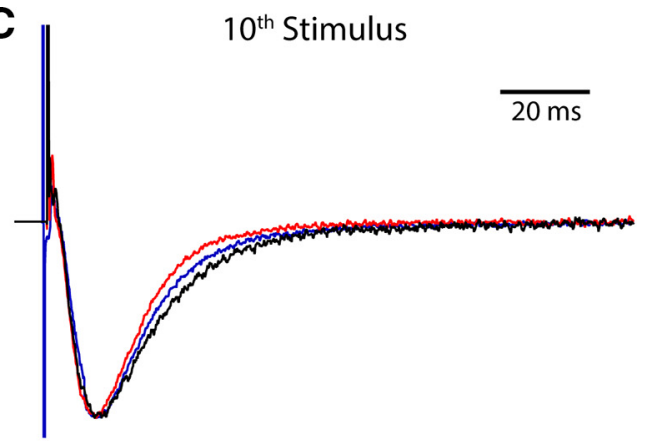

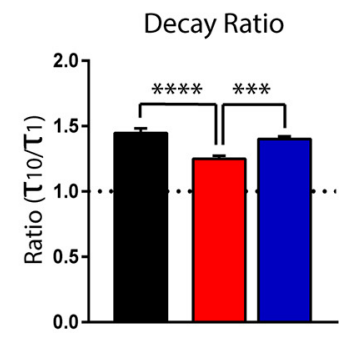

D

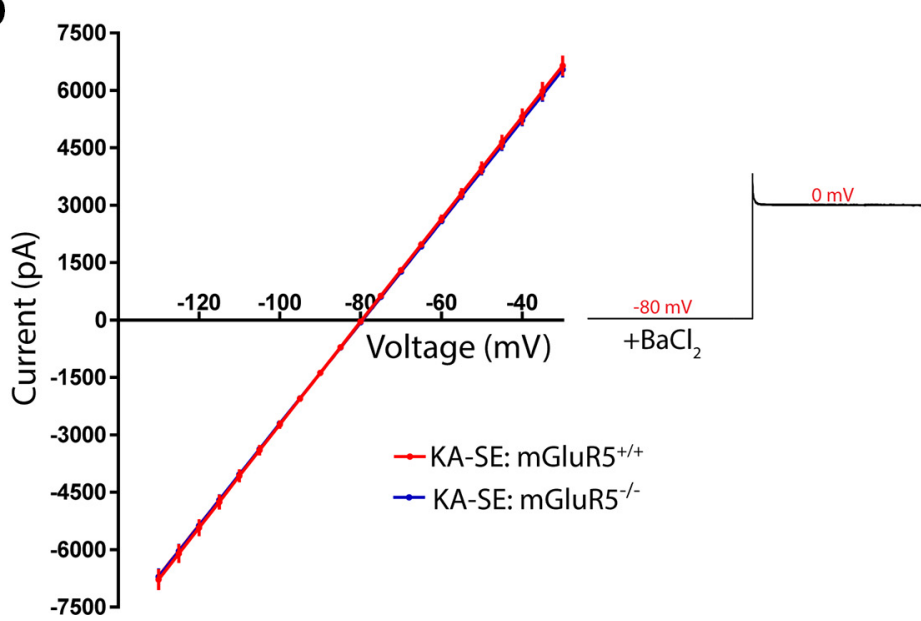

Figure 8. Astrocytes with mGluR5 signaling in the latent period exhibit enhancements in glutamate uptake following high-frequency, synaptic glutamate release. A, A representative GTC from a naive, KA-SE:mGluR5 ${ }^{+/+}$, and KA-SE:mGluR5 ${ }^{-/-}$astrocyte recorded during a $100 \mathrm{~Hz}$ stimulus train ( $100 \mathrm{~ms}, 6 \mathrm{~mA}$ ). Traces were not peak normalized to display differences in facilitation, quantified in the associated bar graph. $\boldsymbol{B}$, The residual current $\left(I_{\text {Res }}\right)$ following the 10th stimulation is compared between groups after peak-amplitude normalization. $\boldsymbol{C}$, A representative GTC response to the 10th stimulus is isolated by subtraction methods, then peak-normalized, and peak-aligned. The first-order $\tau$ decay value following the 10th stimulus is quantified in the associated bar graph along with a comparison of $\tau$ decay ratios following single $\left(\tau_{1}\right)$ and train stimuli $\left(\tau_{10}\right)$. $\boldsymbol{D}$, Current-voltage $(I-\eta)$ curves are plotted for KA-SE:mGluR5 ${ }^{+/+}$and KA-SE:mGluR5 ${ }^{-/-}$ astrocytes as the mean \pm SEM current values across a series of voltage steps. The curve is displayed between -130 to $-30 \mathrm{mV}$ in $5 \mathrm{mV}$ steps. The corresponding holding protocol is displayed in the associated trace. I-V curves were recorded in the presence of $\mathrm{BaCl}_{2}(200 \mu \mathrm{M})$ and indicate a lack of inward rectification. Additionally, the conductance values (slope) obtained from KA-SE: $\mathrm{mGluR5}^{+/+}$and KA-SE:mGluR5 ${ }^{-1-}$ astrocytes are statistically similar. Bar graphs represent group mean \pm SEM values. Group data represent $n=14$ cells from 8 animals in the naive group, $n=$ 14 cells from 5 animals in the KA-SE:mGluR5 ${ }^{+/+}$group, and $n=18$ cells from 6 animals in the KA-SE:mGluR5 ${ }^{-1-}$ group. One-way ANOVA with Tukey's post hoc test: * $p<0.05 ;{ }^{* *} p<0.01$; ${ }^{* * *} p<0.001 ;{ }^{* * *} p<0.0001$.

mGluR5 $^{+/+}$and KA-SE:mGluR5 ${ }^{-/-} ; F_{(2,41)}=9.061$; naive vs KA-SE:mGluR5 ${ }^{+/+}, p=0.0014$; naive vs KA-SE:mGluR5 ${ }^{-/-}$, $p=0.0024$; one-way ANOVA with Tukey's post hoc test; Fig. $8 A$ ). This increased GTC amplitude ratio (10th pulse/first pulse) in the naive condition suggests that greater presynaptic facilitation can occur in naive hippocampal slice during highfrequency activity, which was not evident in paired-pulse studies. Reduced presynaptic facilitation following KA-SE does not appear to be influenced by the selective loss of mGluR5 signaling ( $p=0.8982$, Tukey's post hoc test; Fig. $8 A$ ), with similar facilitation values obtained in $\mathrm{mGluR} 5^{+/+}$and mGluR5 ${ }^{-/-}$slices following KA-SE.

Independent of facilitation, we additionally noted that GTCs recorded in naive and KA-SE:mGluR5 ${ }^{-1-}$ astrocytes had a significantly increased residual current amplitude $\left(\mathrm{I}_{\text {Res }}\right.$; Fig. $\left.8 B\right)$ relative to KA-SE:mGluR5 ${ }^{+/+}$astrocytes. The increased residual current amplitude was not due to differences in GTC peak amplitude (after normalization: $F_{(2,43)}=7.659$; KA-SE:mGluR5 ${ }^{+/+}$ vs naive, $p=0.0011$; KA-SE:mGluR $5^{+/+}$vs KA-SE:mGluR5 ${ }^{-/-}$, $p=0.0333$; one-way ANOVA with Tukey's post hoc test). The residual component of the GTC may represent an important signature of glutamate transport (see Discussion) and does not appear to be confounded by differences in $\mathrm{K}^{+}$conductance. Indeed, recordings are all performed in $\mathrm{BaCl}_{2}(200 \mu \mathrm{M})$ to block the inward rectifying $\mathrm{K}^{+}$conductance in astrocytes. Additionally, whole-cell conductance plots are identical between KA-SE: mGluR5 ${ }^{+/+}$and KA-SE:mGluR5 ${ }^{-/-}$astrocyte recordings $\left(F_{(1,710)}=1.508 ; p=0.2199\right.$, linear regression slope comparison; linear regression goodness of fit: 97.5\%; Fig. 8D).

To determine the efficacy of glutamate uptake following highfrequency stimulation, we determined the rate of GTC decay following the 10th pulse, by fitting a first-order, exponential decay constant $(\tau)$. We observed that astrocytes with mGluR5 signaling in epileptogenesis (KA-SE:mGluR ${ }^{+/+}$) display faster $\tau$ values (Fig. $8 C$ ), relative to both naive astrocytes and astrocytes without mGluR5 signaling in epileptogenesis $\left(\tau: F_{(2,43)}=32.01\right.$; 
vs naive, $p<0.0001$; vs KA-SE:mGluR5 ${ }^{-1-}, p=0.0004$; one-way ANOVA with Tukey's post hoc test; Fig. $8 C$ ). Additionally, astrocytes with mGluR5 signaling display a nearly equivalent rate of GTC decay following a single stimulus and a train of stimuli $\left(\tau 10 / \tau 1\right.$ at $30^{\circ} \mathrm{C}$; Fig. $\left.8 \mathrm{C}\right)$. On the other hand, naive astrocytes and astrocytes without $\mathrm{mGluR} 5$ signaling in epileptogenesis display a $45 \%$ slower rate of GTC decay following a train stimulus relative to a single stimulus $\left(\tau 10 / \tau 1: F_{(2,43)}=13.73\right.$; KA-SE:mGluR5 ${ }^{+/+}$ vs naive, $p<0.0001$; KA-SE:mGluR $5^{+/+}$vs KA-SE:mGluR5 ${ }^{-/-}$, $p=0.0006$; one-way ANOVA with Tukey's post hoc test; Fig. $8 C$ ). Increased decay ratios $(\tau 10 / \tau 1)$ suggest that glutamate transport is more readily overwhelmed during high-frequency stimulation, potentially creating more permissible conditions for extrasynaptic glutamate spillover to occur (Tanaka et al., 2013). In conclusion, we find that astrocytes with mGluR5 signaling in epileptogenesis unequivocally display a faster rate of highfrequency, synaptic glutamate clearance and maintain similar uptake kinetics following low- and high-frequency glutamate release, representing a gain of function in transport over naive and KA-SE:mGluR5 ${ }^{-1-}$ astrocytes.

\section{Discussion}

Herein, we present evidence that astrocyte mGluR5 signaling can occur in the mature hippocampus following KA-SE. Astrocyte mGluR5-mediated calcium signaling can be observed shortly after KA-SE (72 h) and is maintained throughout the seizure-free latent period and into epilepsy. On the other hand, astrocyte mGluR5 expression and function are transient in animals that do not develop epilepsy (28-35 d after KA-SE), suggesting that mGluR5 expression may serve as a potential marker predicting the continuation or resolution of epileptogenesis. Beyond its role as a correlate of epileptogenesis, we find that hippocampal astrocytes with mGluR5 expression in epileptogenesis (KA-SE: mGluR $5^{+/+}$) display calcium transients following the synaptic release of glutamate, a finding suggesting that astrocytes could recapitulate key mGluR5-dependent functions demonstrated in development. Finally, we describe a potential compensatory consequence of astrocyte mGluR5 signaling in epileptogenesis: faster uptake of glutamate and reductions in the residual current amplitude following high-frequency synaptic release. As it relates to astrocyte mGluR5 signaling, these last findings are novel in the context of epileptogenesis and suggest that astrocyte mGluR5 signaling may recapitulate earlier developmental roles in regulating glutamate uptake.

\section{Astrocyte mGluR5 expression and function: considerations and interpretations}

Astrocyte mGluR5 expression is normally confined to the early weeks of life in the rodent brain (Sun et al., 2013; Morel et al., 2014), with similar observations made in human cortex (Sun et al., 2013). However, as it relates to neuropathology, postmortem or resected tissue from patients across a spectrum of refractory epilepsy cases (Aronica et al., 2001, 2003; Boer et al., 2008; Sukigara et al., 2014), including TLE (Tang et al., 2001), can display mGluR5 colocalization with reactive astrocytes in close proximity to the epileptic foci. Astrocyte mGluR5 expression (Aronica et al., 2000; Ulas et al., 2000; Umpierre et al., 2016) and function (Ding et al., 2007; Szokol et al., 2015) have also been reported across multiple animal models of TLE development. In this sense, it would appear that mGluR5 signaling either reemerges in the astrocyte during epilepsy development, or exists at low levels that are greatly augmented by pathology. There is ongoing debate as to whether mGluR5 signaling may truly be absent in mature hip- pocampal astrocytes (Sloan and Barres, 2014; Panatier and Robitaille, 2016), and how this may relate to humans.

The approaches described herein were not intended to answer the question: does mGluR5 signaling exist in the mature hippocampal astrocyte? Instead, our approaches sought to understand the timing of mGluR5 expression and function in the astrocyte through phases of epilepsy development using methodology that would unambiguously provide evidence for that claim. We observe strong astrocyte mGluR5 colocalization 3-7 d after KA-SE (Fig. 1) that temporally coincides with clear calcium responses to DHPG (Fig. 3), using an unbiased approach to analyze calcium activity. While this approach is unlikely to capture subtle calcium activity that others have argued may be missed at the fine processes (Panatier and Robitaille, 2016), we were also largely unable to observe clear DHPG calcium responses in individual, naive astrocytes with focal agonist application $(n=2$ of 61 cells responding to DHPG; Fig. 5), using a high concentration of DHPG $(500 \mu \mathrm{M})$. Considering the prolonged timing of DHPGinduced calcium activity in the astrocyte ( $>10 \mathrm{~s}$ in the soma), reported in our study and by others (Tang et al., 2015), it is unlikely that our $1 \mathrm{~Hz}$ frame rate was insufficient to capture a calcium response. Thus, our results would strongly suggest that mGluR5 signaling is an exceptionally rare feature of naive astrocytes.

\section{Loss of mGluR5 signaling in astrocytes confers a highly selective phenotype in epileptogenesis}

In the present work, we find that loss of astrocyte mGluR5 signaling during epileptogenesis impairs hippocampal glutamate clearance during high-frequency stimulation; however, the loss of mGluR5 signaling does not alter intrinsic cell properties, or glutamate clearance under low-frequency (i.e., single-pulse; Fig. 7) stimulation. Therefore, the loss of mGluR5 signaling appears to confer a highly selective change in astrocyte physiology. In development, mGluR5 signaling has the potential to alter astrocyte glutamate uptake through multiple mechanisms, including increased GLT-1 expression, PKC-dependent phosphorylation, and motility-dependent changes in synaptic contact. These studies also demonstrate that mGluR5-dependent mechanisms are particularly attuned to high-frequency synaptic activity.

We hypothesize that changes in synaptic contact may be the most likely explanation for mGluR5-related enhancements in high-frequency glutamate uptake during epileptogenesis. In the developing astrocyte, attenuation of $\mathrm{IP}_{3}$ signaling, the downstream effector of mGluR5 and other Gq-linked signaling pathways, reduces the number of Schaffer collateral synapses ensheathed by the astrocyte. As a consequence, astrocytes displayed impaired glutamate uptake during high-frequency stimulation without alterations in glutamate uptake following low-frequency stimulation (Tanaka et al., 2013), similar to our observations. Studies in the hypothalamus have demonstrated that greater ensheathment can facilitate the highly localized uptake of glutamate and reduce its extrasynaptic spillover by physically limiting diffusion (Fleming et al., 2011). In this sense, the effects of ensheathment on glutamate transport would most likely be unmasked during train stimuli, which is more likely to produce spillover (Tanaka et al., 2013). Our future studies will assess whether mGluR5 signaling increases the proportion of Schaffer collateral synapses ensheathed by astrocytes during epileptogenesis, potentially placing glutamate transporters nearer to the synapse as an ultrastructural explanation for enhanced highfrequency uptake kinetics. Previous studies in the rat hippocampus demonstrate that $\sim 50 \%$ of Schaffer collateral synapses have a close astrocytic partner (Ventura and Harris, 1999). If mGluR5 
signaling in pathology initiates a "second wave" of ensheathment in the hippocampus, it could provide a key mechanism for regulating glutamate transporter function beyond expression.

Future studies will assess how alterations in astrocyte glutamate transport impact neuropathology and epileptogenesis as a result of mGluR5 signaling loss. The tamoxifen induction protocol used with our molecular genetic approach could effectively remove functional mGluR5 signaling in the majority ( $82 \%)$ of astrocytes that underwent recombination (marked by td-Tomato labeling); however, we estimate from IHC staining that only 50\% of astrocytes in the hippocampus underwent recombination. Future studies will determine a reliable protocol for inducing recombination in the vast majority of astrocytes ( $75 \%$ of the hippocampal population or more) to study how robust loss of astrocyte mGluR5 signaling may impact key network-level outcomes, such as neuronal loss and spontaneous seizure generation.

Dysregulation of glutamate transport has been demonstrated or suggested to play a critical role in multiple pathologies, including Huntington's disease, Fragile X syndrome, and chronic epilepsy (Ueda et al., 2001; Behrens et al., 2002; Pretto et al., 2014; Higashimori et al., 2016). Commonly, glutamate transport dysregulation has been ascribed to changes in GLT-1 expression levels. However, it is unlikely that changes in GLT-1 expression in the low-dose KA-SE model could comprehensively explain our observed differences in single-pulse and high-frequency glutamate transporter decay. Decreases in GLT-1 expression are likely to influence transporter current amplitude but may not impact the kinetics of uptake unless the ratio of GLAST to GLT-1 is altered (Parsons et al., 2016). An emerging literature is investigating whether $\beta$-lactam antibiotics could combat glutamate transporter dysregulation through their demonstrated ability to increase GLT-1 expression (Rothstein et al., 2005). However, one might also consider that transporter expression or density at a single synapse is only one component of overall transporter efficacy. In hypersynchronous pathologies, transporter density at a single synapse may be far less predictive of network-level glutamate regulation than the number of synapses having any apposing transporters at all. In this sense, understanding mechanisms that promote de novo ensheathment of the synapse could serve as a logical and impactful antiepileptogenic strategy.

\section{Implications of the residual current in glutamate transporter recordings}

Group differences in the residual GTC following $100 \mathrm{~Hz}$ stimulation are an unexpected finding in the present work. The residual current in astrocyte recordings is thought to arise from nonKir4.1 potassium channels and persistent GABA transporter currents (Sibille et al., 2014). Similarly, we observe that a residual current persists in GTC recordings after Kir4.1 channel blockade by the presence of $\mathrm{BaCl}_{2}$ (evidenced by the lack of inward rectification in conductance plots; Fig. 8). While we did not test the contributions of non-Kir4.1 potassium channels (such as "leak channels") or persistent GABA transporter currents to the residual current, we can report that the high-affinity glutamate transporter antagonist TFB-TBOA could strongly attenuate the residual component in our recordings (Fig. 7E). Additionally, the leak conductance is equivalent in astrocytes recorded in mGluR5 ${ }^{+/+}$and mGluR5 ${ }^{-1-}$ animals following KA-SE. While further evaluation is necessary, we posit that a significant portion of the GTC residual current may be a signature of protracted glutamate uptake. If the residual current does reflect prolonged glutamate clearance, it could serve as a signature of glutamate spillover and potentially explain the large differ- ences in "residual" current amplitude between groups following high-frequency stimulation. Complimentary approaches, such as iGluSnFR imaging or simultaneous astrocyte-neuron recordings, may aid in better understanding whether the residual current relates to protracted glutamate uptake.

Overall, our study broadens the understanding of how astrocytes may function in epileptogenesis. Previously, only a single study had investigated the effects of astrocyte mGluR5 signaling in epilepsy development and found that mGluR5 signaling contributes to cell death in the hippocampus during status epilepticus (Ding et al., 2007). The pharmacological approaches used in that study could not definitively establish cell type specificity in distinguishing the contributions of neuronal versus astrocytic mGluR5 activity in cell death. Additionally, mGluR5 expression is developmentally repressed in astrocytes after early development (Sun et al., 2013) and is therefore unlikely to participate in network signaling when SE is induced in the adult animal. By contrast, our findings suggest that mGluR5 expression is a delayed response to status epilepticus because clear mGluR5 expression and function occurred $72 \mathrm{~h}$ after status epilepticus and was not clearly evident in the naive state. Critically, we find that astrocytes with mGluR5 signaling in epileptogenesis demonstrate a reduction in glutamate clearance rates when the network is challenged by high-frequency events. This gain in function might predict that an astrocyte with mGluR5 signaling could better sequester glutamate in the hippocampus, potentially attenuating summation as a mechanism for ictogenesis as well as seizure propagation. If so, the expression of mGluR 5 by astrocytes within epileptic foci may represent a compensatory response to glutamate dysregulation, rather than its mediator.

\section{References}

Aronica E, van Vliet EA, Mayboroda OA, Troost D, da Silva FH, Gorter JA (2000) Upregulation of metabotropic glutamate receptor subtype mGluR3 and mGluR5 in reactive astrocytes in a rat model of mesial temporal lobe epilepsy. Eur J Neurosci 12:2333-2344. CrossRef Medline

Aronica E, Yankaya B, Jansen GH, Leenstra S, van Veelen CW, Gorter JA, Troost D (2001) Ionotropic and metabotropic glutamate receptor protein expression in glioneuronal tumours from patients with intractable epilepsy. Neuropathol Appl Neurobiol 27:223-237. CrossRef Medline

Aronica E, Gorter JA, Jansen GH, van Veelen CW, van Rijen PC, Ramkema M, Troost D (2003) Expression and cell distribution of group I and group II metabotropic glutamate receptor subtypes in Taylor-type focal cortical dysplasia. Epilepsia 44:785-795. CrossRef Medline

Behrens PF, Franz P, Woodman B, Lindenberg KS, Landwehrmeyer GB (2002) Impaired glutamate transport and glutamate-glutamine cycling: downstream effects of the Huntington mutation. Brain 125:1908-1922. CrossRef Medline

Bergles DE, Jahr CE (1997) Synaptic activation of glutamate transporters in hippocampal astrocytes. Neuron 19:1297-1308. CrossRef Medline

Bernardinelli Y, Randall J, Janett E, Nikonenko I, König S, Jones EV, Flores CE, Murai KK, Bochet CG, Holtmaat A, Muller D (2014) Activity-dependent structural plasticity of perisynaptic astrocytic domains promotes excitatory synapse stability. Curr Biol 24:1679-1688. CrossRef Medline

Boer K, Troost D, Timmermans W, Gorter JA, Spliet WG, Nellist M, Jansen F, Aronica E (2008) Cellular localization of metabotropic glutamate receptors in cortical tubers and subependymal giant cell tumors of tuberous sclerosis complex. Neuroscience 156:203-215. CrossRef Medline

Bonilla-Del Riotao I, Puente N, Penasco S, Rico I, Gutierrez-Rodriotaguez A, Elezgarai I, Ramos A, Reguero L, Gerrikagoitia I, Christie BR, Nahirney P, Grandes P (2017) Adolescent ethanol intake alters cannabinoid type-1 receptor localization in astrocytes of the adult mouse hippocampus. Addict Biol. Advance online publication. Retrieved November 23, 2017. doi: 10.1111/adb.12585.

Bowser DN, Khakh BS (2004) ATP excites interneurons and astrocytes to increase synaptic inhibition in neuronal networks. J Neurosci 24:86068620. CrossRef Medline

Cai Z, Schools GP, Kimelberg HK (2000) Metabotropic glutamate receptors in 
acutely isolated hippocampal astrocytes: developmental changes of mGluR5 mRNA and functional expression. Glia 29:70-80. CrossRef Medline

Colavita M, Terral G, Lemercier CE, Drago F, Marsicano G, Massa F (2016) Layer-specific potentiation of network GABAergic inhibition in the CA1 area of the hippocampus. Sci Rep 6:28454. CrossRef Medline

Devaraju P, Sun MY, Myers TL, Lauderdale K, Fiacco TA (2013) Astrocytic group I mGluR-dependent potentiation of astrocytic glutamate and potassium uptake. J Neurophysiol 109:2404-2414. CrossRef Medline

Diamond JS, Jahr CE (2000) Synaptically released glutamate does not overwhelm transporters on hippocampal astrocytes during high-frequency stimulation. J Neurophysiol 83:2835-2843. CrossRef Medline

Ding S, Fellin T, Zhu Y, Lee SY, Auberson YP, Meaney DF, Coulter DA, Carmignoto G, Haydon PG (2007) Enhanced astrocytic $\mathrm{Ca}^{2+}$ signals contribute to neuronal excitotoxicity after status epilepticus. J Neurosci 27:10674-10684. CrossRef Medline

Dixon SJ, Yu R, Panupinthu N, Wilson JX (2004) Activation of P2 nucleotide receptors stimulates acid efflux from astrocytes. Glia 47:367-376. CrossRef Medline

Ferraguti F, Shigemoto R (2006) Metabotropic glutamate receptors. Cell Tissue Res 326:483-504. CrossRef Medline

Fleming TM, Scott V, Naskar K, Joe N, Brown CH, Stern JE (2011) Statedependent changes in astrocyte regulation of extrasynaptic NMDA receptor signalling in neurosecretory neurons. J Physiol 589:3929-3941. CrossRef Medline

Gee JM, Smith NA, Fernandez FR, Economo MN, Brunert D, Rothermel M, Morris SC, Talbot A, Palumbos S, Ichida JM, Shepherd JD, West PJ, Wachowiak M, Capecchi MR, Wilcox KS, White JA, Tvrdik P (2014) Imaging activity in neurons and glia with a Polr2a-based and credependent GCaMP5G-IRES-tdTomato reporter mouse. Neuron 83: 1058-1072. CrossRef Medline

Higashimori H, Morel L, Huth J, Lindemann L, Dulla C, Taylor A, Freeman M, Yang Y (2013) Astroglial FMRP-dependent translational down-regulation of mGluR5 underlies glutamate transporter GLT1 dysregulation in the fragile X mouse. Hum Mol Genet 22:2041-2054. CrossRef Medline

Higashimori H, Schin CS, Chiang MS, Morel L, Shoneye TA, Nelson DL, Yang Y (2016) Selective deletion of astroglial FMRP dysregulates glutamate transporter GLT1 and contributes to fragile X syndrome phenotypes in vivo. J Neurosci 36:7079-7094. CrossRef Medline

Honsek SD, Walz C, Kafitz KW, Rose CR (2012) Astrocyte calcium signals at Schaffer collateral to CA1 pyramidal cell synapses correlate with the number of activated synapses but not with synaptic strength. Hippocampus 22:29-42. CrossRef Medline

Lauckner JE, Hille B, Mackie K (2005) The cannabinoid agonist WIN55212-2 increases intracellular calcium via CB1 receptor coupling to Gq/11 G proteins. Proc Natl Acad Sci U S A 102:19144-19149. CrossRef Medline

Marshall JJ, Xu J, Contractor A (2018) Kainate receptors inhibit glutamate release via mobilization of endocannabinoids in striatal direct pathway spiny projection neurons. J Neurosci 38:3901-3910. CrossRef Medline

Morel L, Higashimori H, Tolman M, Yang Y (2014) VGluT1 ${ }^{+}$neuronal glutamatergic signaling regulates postnatal developmental maturation of cortical protoplasmic astroglia. J Neurosci 34:10950-10962. CrossRef Medline

Panatier A, Robitaille R (2016) Astrocytic mGluR5 and the tripartite synapse. Neuroscience 323:29-34. CrossRef Medline

Parsons MP, Vanni MP, Woodard CL, Kang R, Murphy TH, Raymond LA (2016) Real-time imaging of glutamate clearance reveals normal striatal uptake in Huntington disease mouse models. Nat Commun 7:11251. CrossRef Medline

Porter JT, McCarthy KD (1995) Adenosine receptors modulate $\left[\mathrm{Ca}^{2+}\right] \mathrm{i}$ in hippocampal astrocytes in situ. J Neurochem 65:1515-1523. CrossRef Medline

Porter JT, McCarthy KD (1996) Hippocampal astrocytes in situ respond to glutamate released from synaptic terminals. J Neurosci 16:5073-5081. CrossRef Medline

Pretto DI, Kumar M, Cao Z, Cunningham CL, Durbin-Johnson B, Qi L, Berman R, Noctor SC, Hagerman RJ, Pessah IN, Tassone F (2014) Reduced excitatory amino acid transporter 1 and metabotropic glutamate receptor 5 expression in the cerebellum of fragile $\mathrm{X}$ mental retardation gene 1 premutation carriers with fragile $\mathrm{X}$-associated tremor/ataxia syndrome. Neurobiol Aging 35:1189-1197. CrossRef Medline
Racine RJ (1972) Modification of seizure activity by electrical stimulation: II. Motor seizure. Electroencephalogr Clin Neurophysiol 32:281-294. CrossRef Medline

Rothstein JD, Dykes-Hoberg M, Pardo CA, Bristol LA, Jin L, Kuncl RW, Kanai Y, Hediger MA, Wang Y, Schielke JP, Welty DF (1996) Knock-out of glutamate transporters reveals a major role for astroglial transport in excitotoxicity and clearance of glutamate. Neuron 16:675-686. CrossRef Medline

Rothstein JD, Patel S, Regan MR, Haenggeli C, Huang YH, Bergles DE, Jin L, Dykes Hoberg M, Vidensky S, Chung DS, Toan SV, Bruijn LI, Su ZZ, Gupta P, Fisher PB (2005) Beta-lactam antibiotics offer neuroprotection by increasing glutamate transporter expression. Nature 433:73-77. CrossRef Medline

Schreiner AE, Durry S, Aida T, Stock MC, Rüther U, Tanaka K, Rose CR, Kafitz KW (2014) Laminar and subcellular heterogeneity of GLAST and GLT-1 immunoreactivity in the developing postnatal mouse hippocampus. J Comp Neurol 522:204-224. CrossRef Medline

Sibille J, Pannasch U, Rouach N (2014) Astroglial potassium clearance contributes to short-term plasticity of synaptically evoked currents at the tripartite synapse. J Physiol 592:87-102. CrossRef Medline

Sloan SA, Barres BA (2014) Looks can be deceiving: reconsidering the evidence for gliotransmission. Neuron 84:1112-1115. CrossRef Medline

Srinivasan R, Lu TY, Chai H, Xu J, Huang BS, Golshani P, Coppola G, Khakh BS (2016) New transgenic mouselines for selectively targeting astrocytes and studying calcium signals in astrocyte processes in situ and in vivo. Neuron 92:1181-1195. CrossRef Medline

Sukigara S, Dai H, Nabatame S, Otsuki T, Hanai S, Honda R, Saito T, Nakagawa E, Kaido T, Sato N, Kaneko Y, Takahashi A, Sugai K, Saito Y, Sasaki M, Goto Y, Koizumi S, Itoh M (2014) Expression of astrocyte-related receptors in cortical dysplasia with intractable epilepsy. J Neuropathol Exp Neurol 73:798-806. CrossRef Medline

Sun W, McConnell E, Pare JF, Xu Q, Chen M, Peng W, Lovatt D, Han X, Smith Y, Nedergaard M (2013) Glutamate-dependent neuroglial calcium signaling differs between young and adult brain. Science 339:197200. CrossRef Medline

Szokol K, Heuser K, Tang W, Jensen V, Enger R, Bedner P, Steinhäuser C, Taubøll E, Ottersen OP, Nagelhus EA (2015) Augmentation of $\mathrm{Ca}(2+)$ signaling in astrocytic endfeet in the latent phase of temporal lobe epilepsy. Front Cell Neurosci 9:49. CrossRef Medline

Tabatadze N, Huang G, May RM, Jain A, Woolley CS (2015) Sex differences in molecular signaling at inhibitory synapses in the hippocampus. J Neurosci 35:11252-11265. CrossRef Medline

Takahashi DK, Vargas JR, Wilcox KS (2010) Increased coupling and altered glutamate transport currents in astrocytes following kainic-acid-induced status epilepticus. Neurobiol Dis 40:573-585. CrossRef Medline

Tanaka K, Watase K, Manabe T, Yamada K, Watanabe M, Takahashi K, Iwama H, Nishikawa T, Ichihara N, Kikuchi T, Okuyama S, Kawashima N, Hori S, Takimoto M, Wada K (1997) Epilepsy and exacerbation of brain injury in mice lacking the glutamate transporter GLT-1. Science 276:1699-1702. CrossRef Medline

Tanaka M, Shih PY, Gomi H, Yoshida T, Nakai J, Ando R, Furuichi T, Mikoshiba K, Semyanov A, Itohara S (2013) Astrocytic $\mathrm{Ca}^{2+}$ signals are required for the functional integrity of tripartite synapses. Mol Brain 6:6. CrossRef Medline

Tang FR, Lee WL, Yeo TT (2001) Expression of the group I metabotropic glutamate receptor in the hippocampus of patients with mesial temporal lobe epilepsy. J Neurocytol 30:403-411. Medline

Tang W, Szokol K, Jensen V, Enger R, Trivedi CA, Hvalby Ø, Helm PJ, Looger LL, Sprengel R, Nagelhus EA (2015) Stimulation-evoked $\mathrm{Ca}^{2+}$ signals in astrocytic processes at hippocampal CA3-CA1 synapses of adult mice are modulated by glutamate and ATP. J Neurosci 35:3016-3021. CrossRef Medline

Thomson KE, White HS (2014) A novel open-source drug-delivery system that allows for first-of-kind simulation of nonadherence to pharmacological interventions in animal disease models. J Neurosci Methods 238:105111. CrossRef Medline

Tse K, Puttachary S, Beamer E, Sills GJ, Thippeswamy T (2014) Advantages of repeated low dose against single high dose of kainate in C57BL/6J mouse model of status epilepticus: behavioral and electroencephalographic studies. PLoS One 9:e96622. CrossRef Medline 
Ueda Y, Doi T, Tokumaru J, Yokoyama H, Nakajima A, Mitsuyama Y, Ohya-Nishiguchi H, Kamada H, Willmore LJ (2001) Collapse of extracellular glutamate regulation during epileptogenesis: downregulation and functional failure of glutamate transporter function in rats with chronic seizures induced by kainic acid. J Neurochem 76: 892-900. CrossRef Medline

Ulas J, Satou T, Ivins KJ, Kesslak JP, Cotman CW, Balázs R (2000) Expression of metabotropic glutamate receptor 5 is increased in astrocytes after kainate-induced epileptic seizures. Glia 30:352-361. CrossRef Medline

Umpierre AD, Bennett IV, Nebeker LD, Newell TG, Tian BB, Thomson KE, White HS, White JA, Wilcox KS (2016) Repeated low-dose kainate administration in C57BL/6 mice produces temporal lobe epilepsy pathol- ogy but infrequent spontaneous seizures. Exp Neurol 279:116-126. CrossRef Medline

Vargas JR, Takahashi DK, Thomson KE, Wilcox KS (2013) The expression of kainate receptor subunits in hippocampal astrocytes after experimentally induced status epilepticus. J Neuropathol Exp Neurol 72:919-932. CrossRef Medline

Ventura R, Harris KM (1999) Three-dimensional relationships between hippocampal synapses and astrocytes. J Neurosci 19:6897-6906. CrossRef Medline

Xu J, Zhu Y, Contractor A, Heinemann SF (2009) mGluR5 has a critical role in inhibitory learning. J Neurosci 29:3676-3684. CrossRef Medline 